\title{
Multidimensional Multirate Filters and Filter Banks Derived from One-Dimensional Filters
}

\author{
Tsuhan Chen, Student Member, IEEE, and P. P. Vaidyanathan, Fellow, IEEE
}

\begin{abstract}
We present a method by which every multidimensional (MD) filter with an arbitrary parallelepiped-shaped passband support can be designed and implemented efficiently. We show that all such filters can be designed starting from an appropriate one-dimensional prototype filter and performing a simple transformation. With $D$ denoting the number of dimensions, we hence reduce the complexity of design as well as implementation of the MD filter from $\mathcal{O}\left(N^{D}\right)$ to $\mathcal{O}(N)$. Furthermore, by using the polyphase technique, we can obtain an implementation with complexity of only $2 \mathrm{~N}$ in the two-dimensional special case. With our method, the Nyquist constraint and zero-phase requirement can be satisfied easily. In the IIR case, stability of the designed filters is also easily achieved. Even though the designed filters are in general nonseparable, these filters have separable polyphase components. One special application of this method is in MD multirate signal processing, where filters with parallelepiped-shaped passbands are used in decimation, interpolation, and filter banks. Some generalizations and other applications of this approach, including MD uniform DFT quadrature mirror filter banks which achieve perfect reconstruction, are studied. Several design examples are also given.
\end{abstract}

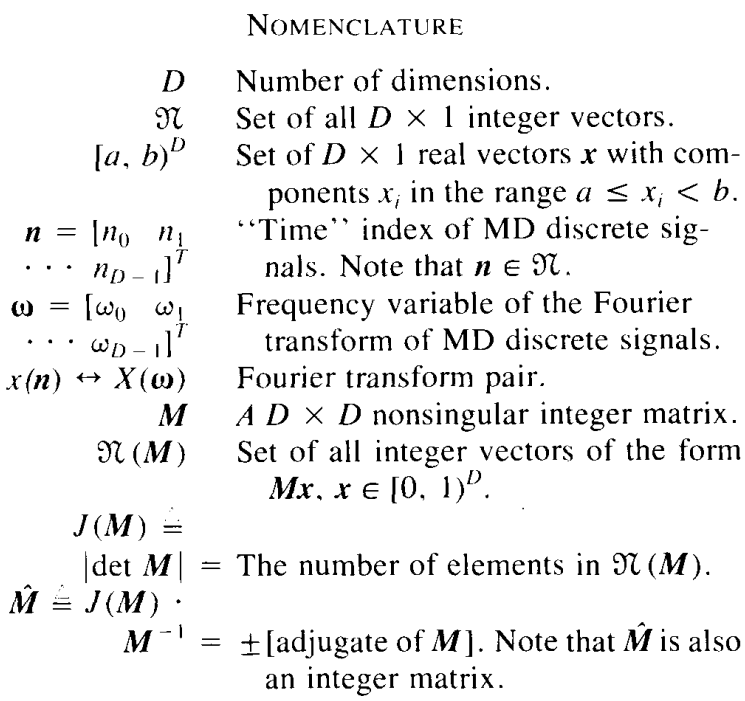

Manuseript received February 9, 1991: revised July 21. 1992. The associate editor coordinating the review of this paper and approving it for publication was Prof. Faye Boudreaux-Bartels. This work was supported in part by the National Science Foundation Grants MIP 8604456, MIP 8919196 . and by matching funds from Tektronix. Inc.. Hughes Aircraft Company, and Rockwell International.

The authors are with the Department of Electrical Engineering. Califor nia Institute of Technology. Pasadena. CA 91125

IEEE Log Number 9207535

\author{
SPD $(\boldsymbol{V})$ \\ (symmetric \\ Set of all real vectors of the form \\ parallel-

$$
\boldsymbol{V} \boldsymbol{x}, \boldsymbol{x} \in[-1,1)^{D} \text {. }
$$

epiped of $\boldsymbol{V}$ )

\section{INTRODUCTION}

$\mathrm{R}$ ECENTLY, there has been much research work on multidimensional (MD) multirate techniques which have found applications in subband coding of images and video data. In this field, MD filters with parallelepipedshaped passbands are used commonly, especially as decimation filters and interpolation filters. For various reasons [1], both the design and implementation of such filters are more complex than in the one-dimensional (ID) case. In the two-dimensional (2D) case, some authors have proposed efficient techniques to design diamond filters, fan filters, and directional filters, by starting from a 1D prototype [2]-[6]. While these methods are very valuable (both for design and implementation) as shown in these references, they do not place in evidence a technique for generalization to arbitrary dimensions and arbitrary parallelepiped-shaped passbands. In this paper we will present a method which works for arbitrary dimensions and arbitrary parallelepiped-shaped passbands. For example, given the decimation/expansion matrix $\boldsymbol{M}$, we first design a $1 \mathrm{D}$ low-pass prototype filter $p(n)$ with passband cutoff frequency at $\pi /|\operatorname{det} \boldsymbol{M}|$. We then define the impulse response $h(\boldsymbol{n})$ of the MD filter as follows ( $D$ is the number of dimensions):

Step 1. First define the MD separable filter $h^{(s)}(n) \triangleq$ $p\left(n_{0}\right) p\left(n_{1}\right) \cdots p\left(n_{D-1}\right)$.

Step 2. Then define $h(\boldsymbol{n})=c_{0} h^{(s)}(\hat{\boldsymbol{M}} \boldsymbol{n})$, where $\hat{\boldsymbol{M}}=$ \pm [adjugate of $\boldsymbol{M}$ ] and $c_{0}$ is some scale factor.

The claim is that the resulting filter $h(\boldsymbol{n})$ is low pass with the passband support in

$$
\omega=\pi M^{-T} x, \quad x \in[-1,1)^{D}
$$

as is the case in many of the 2D designs of [2]-[6]. Even though $h(n)$ is in general not separable, it can be considered as a generalization of separable filters in the sense that we can derive it by decimating a separable filter $h^{(s)}(\boldsymbol{n})$. Also, as shown later, the polyphase components of $h(n)$ are indeed separable.

Note that the region defined in (1.1) is not an arbitrary parallelepiped, but governed by the integer matrix $\boldsymbol{M}$. To 
represent an arbitrary parallelepiped, we need to use $\pi \boldsymbol{H}^{-T} \boldsymbol{x}$, where $\boldsymbol{x} \in[-1,1)^{D}$ and $\boldsymbol{H}$ is a nonsingular matrix with rational elements. (The irrational case can be approximated by a rational matrix $\boldsymbol{H}$.) In Sections II and III, we will state in details the general procedure for designing filters with such arbitrary parallelepiped-shaped passband supports.

The above design rule and its proof are the same for any parallelepiped-shaped passbands, and for any number of dimensions. In this respect the method differs from, and generalizes, earlier ones. Note that the 1D prototype filter depends only on $|\operatorname{det} \boldsymbol{M}|$. As done in [4] and [6], we also provide bounds on the passband and stopband ripples of the resulting MD filter in terms of the ripples of the $1 \mathrm{D}$ prototype. We will also exploit the above relation between ID and MD filters to obtain efficient polyphase implementations (Section IV). Preliminary (less detailed versions) of our results have been reported earlier in [7].

With our method, the Nyquist constraint and zero-phase requirement can be satisfied easily. Also, the designed filters all have separable polyphase components. In the IIR case, the stability of the resulting filters is also guaranteed. In addition to filters with parallelepiped-shaped passbands, many other filters can be designed by minor combinations of these filters (Section V). Because our method results in a close relation between the polyphase components of the $1 \mathrm{D}$ prototype and those of the resulting MD filter, the design and implementation of MD uniform DFT quadrature mirror filter (QMF) banks can be simplified (Section VI). Many useful MD DFT QMF banks, including those that achieve perfect reconstruction (PR), are presented, together with several design examples. We will also make some comments about how to deal with the case when the passband support is very small (Section VII).

Notations: Boldfaced letters denote matrices and vectors. The symbol $\boldsymbol{I}$ is reserved for the identity matrix. The notations $\boldsymbol{A}^{T}, \boldsymbol{A}^{*}$, and $\boldsymbol{A}^{-T}$ denote the transpose, the conjugate, and the inverse transpose of $\boldsymbol{A}$, respectively. The row and column indices typically begin from zero. $D$ denotes the number of dimensions, $\mathfrak{N}$ stands for the set of all $D \times 1$ integer vectors, and $[a, b)^{D}$ is the set of $D \times 1$ real vectors $x$ with components $x_{i}$ in the range $a \leq x_{i}<$ $b$. Let $\boldsymbol{n}=\left[\begin{array}{llll}n_{0} & n_{1} & \cdots & n_{D-1}\end{array}\right]^{T}$ denote the "time" index of MD discrete signals. All $n_{i}$ 's are integer, so that $n \in$ $\Re$. Also, $\omega=\left[\begin{array}{lll}\omega_{0} & \omega_{1} \cdots & \cdots \omega_{D-1}\end{array}\right]^{T}$ is the frequency variable of the Fourier transform of MD discrete signals. The Fourier transform pair, $x(\boldsymbol{n}) \leftrightarrow X(\boldsymbol{\omega})$, is defined as

$$
\begin{aligned}
& X(\omega)=\sum_{n \in \mathfrak{N}} x(\boldsymbol{n}) e^{-j \omega^{T} n}, \\
& x(n)=\frac{1}{(2 \pi)^{D}} \int_{\omega \in[-\pi, \pi)^{D}} X(\boldsymbol{\omega}) e^{j \omega^{T} n} d \omega .
\end{aligned}
$$

Refer to [1], [8]-[13] for other preliminaries of MD multirate signal processing. An excellent recent reference on MD multirate systems is [12], which also introduces and reviews many notations we have used. Some of the notations we use here are slightly modified versions of those in [12], and suit our discussions better. Although we will define each new notation as we proceed, all of these notations are summarized in the Nomenclature section for quick reference.

\section{The Impulse Response of an IdeAl Low-Pass FILTER}

In this section we give the key equation which can be considered to be the theoretical foundation for the rest of the paper. Let $\boldsymbol{M}$ be a $D \times D$ nonsingular integer matrix. The $\boldsymbol{M}$-fold decimated version of $\boldsymbol{x}(\boldsymbol{n})$ is defined as $y(\boldsymbol{n})$ $=x(M n)$, with the frequency domain relation [11], [12]

$$
Y(\omega)=\frac{1}{J(M)} \sum_{k \in \mathfrak{R}\left(M^{T}\right)} X\left(M^{-T}(\omega-2 \pi k)\right)
$$

where $J(\boldsymbol{M})$ denotes $|\operatorname{det} \boldsymbol{M}|$, and $\mathfrak{T}\left(\boldsymbol{M}^{T}\right)$ denotes the set of all integer vectors of the form $\boldsymbol{M}^{T} \boldsymbol{x}$ for $\boldsymbol{x} \in[0,1)^{D}$. It can be shown that the number of elements in either $\mathscr{N}(M)$ or $\mathscr{N}\left(\boldsymbol{M}^{T}\right)$ is $J(\boldsymbol{M})$.

The $\boldsymbol{M}$-fold expanded version of $\boldsymbol{x}(\boldsymbol{n})$ is defined as

$$
y(n)= \begin{cases}x\left(M^{-1} n\right) & M^{-1} n \in \mathfrak{X} \\ 0 & \text { otherwise }\end{cases}
$$

so that $Y(\omega)=X\left(M^{T} \omega\right)$ [11], [12]. To prevent aliasing due to the $\boldsymbol{M}$-fold decimation (or eliminate images in the $\boldsymbol{M}$-fold expansion), a decimation (or interpolation) filter $H(\omega)$ is necessary. Typically, this filter has a parallelepiped-shaped passband support in

$$
\boldsymbol{\omega}=\pi \boldsymbol{M}^{-T} \boldsymbol{x}+2 \pi \boldsymbol{k}, \quad \boldsymbol{x} \in[-1,1)^{D}, \quad k \in \mathfrak{N} .
$$

For example, in the $2 \mathrm{D}$ case, the region $\pi \boldsymbol{M}^{-T} \boldsymbol{x}, \boldsymbol{x} \in[-1$, $1)^{2}$, denotes (in terms of the frequencies $\omega_{0}$ and $\omega_{1}$ )

$$
\begin{aligned}
& -\pi \leq M_{00} \omega_{0}+M_{10} \omega_{1} \leq \pi, \\
& -\pi \leq M_{01} \omega_{0}+M_{11} \omega_{1}<\pi
\end{aligned}
$$

and is used in [2]-[6]. Fig. 1 shows this region for

$$
\boldsymbol{M}=\left[\begin{array}{rr}
1 & -1 \\
1 & 2
\end{array}\right]
$$

We will now use an abbreviated notation to indicate (2.3). We use the notation SPD $(\boldsymbol{V})$ (symmetric parallelepiped generated by $\boldsymbol{V}$ ) to denote the set of all real vectors of the form $\boldsymbol{V} \boldsymbol{x}$, for $\boldsymbol{x} \in[-1,1)^{D}$. We can then rewrite (2.3) as

$$
\operatorname{SPD}\left(\pi M^{-T}\right)+2 \pi k, \quad k \in \Re .
$$

The terms $2 \pi k$ shows that SPD $\left(\pi M^{-T}\right)$ is repeated every $2 \pi$ in each dimension. We often say that the passband support of the filter is given by $\operatorname{SPD}\left(\pi M^{-T}\right)$, and take the periodicity for granted, i.e., do not show the term $2 \pi k$ explicitly.

Let $H(\omega)$ denote the frequency response of an ideal low-pass decimation/interpolation filter for the $\boldsymbol{M}$-fold 


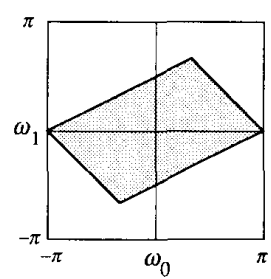

Fig. 1. Typical passband of multirate filters

decimator/expander. The passband support is as in (2.3) or $(2.5)$, i.e.,

$$
H(\omega)= \begin{cases}1 & \text { if } \omega \in \operatorname{SPD}\left(\pi M^{-T}\right) \\ 0 & \text { otherwise. }\end{cases}
$$

Let $h(\boldsymbol{n})$ denote the impulse response of $H(\boldsymbol{\omega})$. We now obtain an expression for $h(\boldsymbol{n})$. This expression will reveal a fundamental relation to $1 \mathrm{D}$ low-pass filters, and enable us to design $H(\omega)$ starting from a $1 \mathrm{D}$ prototype. Using the inverse Fourier transform relation in (1.2), we obtain

$$
\begin{aligned}
& h(n)=\frac{1}{(2 \pi)^{D}} \int_{\omega \in \operatorname{SPD}(\pi \cdot M \cdot T)} e^{j \omega^{T} n} d \omega \\
& =\frac{1}{2^{D} J(M)} \int_{x \in[-1,1)^{D}} e^{j \pi x^{T} M \ln } d x \\
& \left(\omega=\pi M^{-T} x\right) \\
& =\frac{1}{2^{D} J(M)} \int_{x \in(-1.1)^{D}} e^{j \pi x^{T} m} d x \\
& \left(\boldsymbol{m}=M^{-1} n\right) \\
& =\frac{1}{2^{D} J(\boldsymbol{M})} \prod_{i=0}^{D-1} \int_{x_{i}=-1}^{1} e^{j \pi m_{i} x_{i}} d x_{i}
\end{aligned}
$$

where $m_{i}$ are the components of the $D \times 1$ vector

$$
m=M^{-1} n=\frac{\hat{M} n}{J(M)}
$$

with $\hat{\boldsymbol{M}}=J(\boldsymbol{M}) \cdot \boldsymbol{M}^{-1}= \pm$ [adjugate of $\boldsymbol{M}$ ]. Evidently, $\hat{\boldsymbol{M}}$ is also an integer matrix. Note, however, that $\boldsymbol{m}$ is a real (not an integer) vector. From the above we obtain

$$
h(\boldsymbol{n})=\frac{1}{J(\boldsymbol{M})} \prod_{i=0}^{D-1} \frac{\sin \left(\pi m_{i}\right)}{\pi m_{i}}
$$

\section{A. Relationship to $1 D$ Filters}

Consider a one-dimensional ideal filter with frequency response $P(\omega)$ as shown in Fig. 2. Its impulse response is given by

$$
p(n)=\frac{\sin \left(\frac{\pi n}{J(\boldsymbol{M})}\right)}{\pi n} .
$$

Starting from this prototype filter $P(\omega)$, suppose we define the $D$-dimensional filter

$$
H^{(s)}(\boldsymbol{\omega})=P\left(\omega_{0}\right) P\left(\omega_{1}\right) \cdots P\left(\omega_{D-1}\right) .
$$

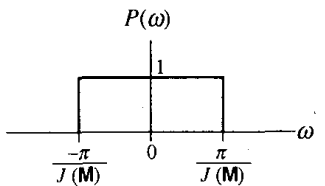

Fig. 2. Frequency response of an ideal low-pass filter

This is a separable low-pass filter, with passband support SPD $(\pi I / J(M))$. Its impulse response is

$$
h^{(s)}(\boldsymbol{n})=p\left(n_{0}\right) p\left(n_{1}\right) \cdots p\left(n_{D-1}\right)
$$

i.e.,

$$
h^{(s)}(\boldsymbol{n})=\prod_{i=0}^{D-1} \frac{\sin \left(\frac{\pi n_{i}}{J(\boldsymbol{M})}\right)}{\pi n_{i}} .
$$

Now consider the quantity $h^{(s)}(\hat{\boldsymbol{M}} \boldsymbol{n})$, which is the $\hat{\boldsymbol{M}}$-fold decimated version of $h^{(*)}(\boldsymbol{n})$. Since $\hat{\boldsymbol{M}} \boldsymbol{n}=J(\boldsymbol{M}) \boldsymbol{M}^{-1} \boldsymbol{n}=$ $J(\boldsymbol{M}) \boldsymbol{m}$, we get

$$
\begin{aligned}
h^{(s)}(\hat{\boldsymbol{M}} \boldsymbol{n})=h^{(s)}(J(\boldsymbol{M}) \boldsymbol{m}) & =\prod_{i=0}^{D-1} \frac{\sin \left(\pi m_{i}\right)}{\pi J(\boldsymbol{M}) m_{i}} \\
& =\frac{1}{J(\boldsymbol{M})^{D}} \prod_{i=0}^{D-1} \frac{\sin \left(\pi m_{i}\right)}{\pi m_{i}} .
\end{aligned}
$$

Comparing (2.14) with (2.9), we obtain the following very simple relation between $h(\boldsymbol{n})$ and $h^{(\cdot)}(\hat{\boldsymbol{M}} \boldsymbol{n})$ :

$$
h(n)=c_{0} h^{(s)}(\hat{M} n)
$$

where $c_{0}=[J(\boldsymbol{M})]^{D-1}=J(\hat{\boldsymbol{M}})$. In other words, $h(\boldsymbol{n})$ is obtained simply by $\hat{\boldsymbol{M}}$-fold decimation of the $D$-dimensional separable sequence $h^{(\cdot)}(\boldsymbol{n})$, followed by scaling with $c_{0} !$

\section{B. Rational Case}

We will generalize this result to ideal filters with the passband support in SPD $\left(\pi \boldsymbol{H}^{-T}\right)$, where $\boldsymbol{H}$ is a $D \times D$ nonsingular matrix with rational elements. Because any irrational matrix can be approximated by rational matrices, this covers any parallelepiped-shaped passband support.

Consider an ideal low-pass filter which has the passband region in SPD $\left(\pi \boldsymbol{H}^{-T}\right)$, i.e.,

$$
G(\omega)= \begin{cases}1 & \text { if } \omega \in \operatorname{SPD}\left(\pi \boldsymbol{H}^{-T}\right) \\ 0 & \text { otherwise. }\end{cases}
$$

Similarly as in (2.7)-(2.9), we can obtain the inverse Fourier transform of $G(\omega)$

$$
g(\boldsymbol{n})=\frac{1}{J(\boldsymbol{H})} \prod_{i=0}^{D-1} \frac{\sin \left(\pi q_{i}\right)}{\pi q_{i}}
$$

where $\boldsymbol{q}=\left[\begin{array}{lll}q_{0} & \cdots & q_{D-1}\end{array}\right]^{T}=\boldsymbol{H}^{-1} \boldsymbol{n}$. Meanwhile, by using the so-called matrix fraction description (MFD), any nonsingular rational matrix can be expressed in its left MFD as $\boldsymbol{H}=\boldsymbol{L}^{-1} \boldsymbol{M}$, where $\boldsymbol{L}$ and $\boldsymbol{M}$ are some nonsin- 
gular integer matrices [14]-[16]. Let us consider $h(L n)$, the $L$-fold decimated version of $h(n)$ in (2.9). We get

$$
\begin{aligned}
& h(\boldsymbol{L n})=\frac{1}{J(\boldsymbol{M})} \prod_{i=0}^{D-1} \frac{\sin \left(\pi q_{i}\right)}{\pi q_{i}}, \\
& \text { where } \boldsymbol{q}=\boldsymbol{M}^{-1} \boldsymbol{L n}=\boldsymbol{H}^{-1} \boldsymbol{n} .
\end{aligned}
$$

Comparing (2.17) and (2.18), we obtain the following relation between $h(\boldsymbol{n})$ and $g(\boldsymbol{n})$ :

$$
g(\boldsymbol{n})=c_{1} h(\boldsymbol{L n})
$$

where $c_{1}=J(\boldsymbol{M}) / J(\boldsymbol{H})=J(\boldsymbol{L})$. In other words, $g(\boldsymbol{n})$ is obtained simply by $L$-fold decimation of $h(n)$, followed by scaling with $c_{1}$.

\section{Design Procedure}

Motivated by the conclusion in Section II, we can design an MD filter with the passband support SPD $\left(\pi \boldsymbol{H}^{-T}\right)$ as follows:

Step 1. Find a left MFD of $\boldsymbol{H}$, say $\boldsymbol{H}=\boldsymbol{L}^{-1} \boldsymbol{M}$.

Step 2. Design a 1D low-pass prototype filter $p(n)$, which can have either finite impulse response (FIR) or infinite impulse response (IIR), with passband region $[-\pi / J(\boldsymbol{M}), \pi / J(\boldsymbol{M}))$.

Step 3. Construct the separable MD filter $h^{(s)}(\boldsymbol{n})$ from $p(n)$ as

$$
h^{(s)}(\boldsymbol{n})=p\left(n_{0}\right) p\left(n_{1}\right) \cdots p\left(n_{D-1}\right) .
$$

Step 4. Define $h(\boldsymbol{n}) \triangleq c_{0} h^{(s)}(\hat{\boldsymbol{M}} \boldsymbol{n})$, where $c_{0}=J(\hat{\boldsymbol{M}})$.

Step 5. Define $g(\boldsymbol{n}) \triangleq c_{1} h(\boldsymbol{L n})$, where $c_{1}=J(\boldsymbol{L})$.

Note that steps 4 and 5 can be combined as one step: $g(\boldsymbol{n}) \triangleq c h^{(s)}(\hat{\boldsymbol{M}} \boldsymbol{L} n)$, where $c=c_{0} c_{1}=J(\hat{\boldsymbol{M}} L)$. Also, when $\boldsymbol{H}$ is itself an integer matrix (so $\boldsymbol{L}$ can be chosen as an identity matrix), we can omit step 5 and simply use the resulting filter $H(\omega)$ of step 4 .

\section{Remarks on the Choice of $\boldsymbol{M}$ and $L$ :}

1) Since we decimate $h(n)$ by a factor to obtain $g(\boldsymbol{n})$, there is a "design overhead factor" of $J(L)$. To reduce such overhead, we need to find a left MFD of $\boldsymbol{H}$ where $\boldsymbol{L}$ has the smallest absolute determinant. For this purpose, it turns out that we should choose the so-called irreducible left MFD of $\boldsymbol{H}$, which is a left MFD where $\boldsymbol{M}$ and $\boldsymbol{L}$ are left coprime [14]-[16].

2) Instead of choosing an irreducible MFD, we can choose an MFD where $\boldsymbol{M}$ is diagonal. This is always possible because $\boldsymbol{H}^{-1}=\boldsymbol{M}^{-1} \boldsymbol{L}$ and we can simply let $m_{i i}$ (the diagonal elements of $\boldsymbol{M}$ ) be the least common multiple of denominators in the $i$ th row of $\boldsymbol{H}^{-1}$. The advantage of diagonal $\boldsymbol{M}$ is that the design of $h(n)$ becomes trivial since it can be done separately in each dimension. However, we pay the expense that the overhead factor $J(L)$ may be higher than the one in an irreducible MFD. Therefore, whether diagonal $\boldsymbol{M}$ or irreducible MFD should be used depends on the matrix $\boldsymbol{H}$.

Because $H^{(s)}(\omega)$ is not ideal, decimation operations in steps 4 and 5 cause some aliasing, both in the passband and the stopband. We now proceed to analyze these ripple sizes, and obtain bounds in the same way as done in [4] and [6]. Suppose the prototype filter $P(\omega)$ has passband ripple $\delta_{1}$ and stopband ripple $\delta_{2}$. Then, it is clear that the frequency response of $H^{(s)}(\omega)$ will satisfy

$$
\begin{gathered}
\left(1-\delta_{1}\right)^{D} \leq\left|H^{(s)}(\omega)\right| \leq\left(1+\delta_{1}\right)^{D} \\
\text { in the passband } \\
0 \leq\left|H^{(s)}(\omega)\right| \leq\left(1+\delta_{1}\right)^{D-1} \delta_{2} \\
\text { in the stopband. }
\end{gathered}
$$

When $\delta_{1}, \delta_{2} \ll 1$, we have

$$
\begin{aligned}
\left(1 \pm \delta_{1}\right)^{D} & \approx 1 \pm D \delta_{1} \\
\text { and } \quad\left(1+\delta_{1}\right)^{D-1} \delta_{2} & \approx \delta_{2} .
\end{aligned}
$$

Therefore, the passband and stopband ripples of $H^{(s)}(\omega)$ are approximately $D \delta_{1}$ and $\delta_{2}$, respectively. Since $h(\boldsymbol{n})=$ $J(\hat{\boldsymbol{M}}) h^{(s)}(\hat{\boldsymbol{M}} \boldsymbol{n})$, from (2.1) we obtain

$$
H(\boldsymbol{\omega})=\sum_{\boldsymbol{k} \in \mathfrak{\mathfrak { M }}\left(\hat{\boldsymbol{M}}^{7}\right)} H^{(\boldsymbol{s})}\left(\hat{\boldsymbol{M}}^{-T}(\boldsymbol{\omega}-2 \pi \boldsymbol{k})\right)
$$

We can see that $H(\omega)$ is the sum of the "stretched" version $H^{(s)}\left(\hat{M}^{-T} \omega\right)$ and $J(\hat{M})-1$ "shifted" versions of it. Therefore, the passband ripple of $H(\omega)$ is at most the sum of one passband ripple and $J(\hat{\boldsymbol{M}})-1$ stopband ripples of $H^{(s)}(\omega)$, and the stopband ripple of $H(\omega)$ is at most the sum of $J(\hat{M})$ stopband ripples of $H^{(i)}(\omega)$. Hence, the peak passband and stopband ripples of the resulting filter $H(\omega)$ should be upper bounded by

$$
\begin{array}{r}
\delta_{1, h}=(J(\hat{\boldsymbol{M}})-1) \delta_{2}+D \delta_{1}, \\
\text { and } \delta_{2, h}=J(\hat{\boldsymbol{M}}) \delta_{2} .
\end{array}
$$

Similarly, we can obtain that the resulting passband and stopband ripple sizes of $g(\boldsymbol{n})$ are upper bounded by

$$
\begin{array}{r}
\tilde{\delta}_{1}=(J(\boldsymbol{L})-1) \delta_{2 . h}+\delta_{1, h} \\
\text { and } \tilde{\delta}_{2}=J(\boldsymbol{L}) \delta_{2, h} .
\end{array}
$$

Note that the resulting ripples generally will be less than these upper bounds. To design $G(\omega)$, we simply choose $\delta_{1}$ and $\delta_{2}$ small enough so that the resulting $G(\omega)$ satisfies the specifications.

\section{Remarks:}

1) Similar bounds of ripple sizes for the case of $2 D$ diamond filters and directional filters can be found in [4] and [6].

2) The technique of transforming a 2D filter with separable rectangular passband into some particular shapes by a change of variables has been mentioned in [2]-[6]. However, our method presents the required prototype filter and the required transform (namely, decimation by $\hat{\boldsymbol{M}} L$ ) for any number of dimensions and any parallelepiped-shaped passband supports. 


\section{Design Example I}

We choose

$$
\boldsymbol{H}=\left|\begin{array}{rr}
1 & -1 \\
1 & 2
\end{array}\right| \text {. }
$$

The desired passband support is same as in Fig. 1. Because $\boldsymbol{H}$ is itself an integer matrix, step 5 can be omitted and $H(\omega)$ will be the desired filter. We use linear programming [17, sect. 3.19] to design the prototype filter $P(\omega)$, a zero-phase Nyquist FIR filter with length $N=$ 59 , passband ripple $\delta_{1}=0.01994$, and stopband ripple $\delta_{2}$ $=0.00888$ (i.e., stopband attenuation $A_{s}=-41.03 \mathrm{~dB}$ ), shown in Fig. 3(a). Following the steps described previously, we obtain $H^{(s)}(\omega)$ and $H(\omega)$, as shown in Figs. 3 (b) and (c). The resulting $H(\omega)$ has passband ripple $\delta_{1}^{\prime}$ $=0.03931$ and stopband ripple $\delta_{2}^{\prime}=0.01778(-35.00$ $\mathrm{dB})$, which are indeed smaller than the estimated values $\left(\delta_{l, h}=0.05765, \delta_{2 . h}=0.02664\right)$ given in (3.5).

\section{Design Example 2}

Suppose we want to design a filter $g(\boldsymbol{n})$ which has passband in the shaded region of Fig. 4(a). This region can be expressed as SPD $\left(\pi \boldsymbol{H}^{-T}\right)$, where

$$
\boldsymbol{H}=\left[\begin{array}{rr}
3 / 5 & -6 / 5 \\
6 / 5 & 3 / 5
\end{array}\right]=\underbrace{\left[\begin{array}{rr}
1 & 2 \\
-2 & 1
\end{array}\right]^{-1}}_{L} \underbrace{\left[\begin{array}{ll}
3 & 0 \\
0 & 3
\end{array}\right]}_{M} .
$$

(The computation of irreducible MFD's can be found in [13]-[15].) For this case, the matrix $\boldsymbol{M}$ turns out to be diagonal and we can utilize the filter $h^{(s)}(n)$ in design example 1 . This filter is decimated by $L$ and scaled by $J(L)$ to obtain $g(\boldsymbol{n})$. The resulting frequency response $G(\boldsymbol{\omega})$, which has passband ripple size $\delta_{1}^{\prime}=0.02719$ and stopband ripple size $\delta_{2}^{\prime}=0.03038(-30.35 \mathrm{~dB})$, is shown in Fig. 4(b).

Preservation of the Zero-Phase Property: A filter having purely real frequency response is called a zero-phase filter. The time domain requirement for zero-phase filters is $f(\boldsymbol{n})=f^{*}(-\boldsymbol{n})[1$, p. 113]. Zero-phase filters introduce no phase distortion, and this is important in many image processing applications. Our filter design method preserves the zero-phase property, i.e., if the ID filter has zero phase, the resulting MD filter $G(\omega)$ also has zero phase. To show this, suppose the ID prototype $p(n)$ has zero phase, i.e., $p(n)=p^{*}(-n)$. From (3.1), we know $h^{(s)}(\boldsymbol{n})=h^{(s)^{*}}(-\boldsymbol{n})$. Then, $g(\boldsymbol{n})=c h^{(s)}(\hat{M} L \boldsymbol{n})=$ $c h^{(s)^{*}}(-\hat{\boldsymbol{M}} \boldsymbol{L n})=g^{*}(-\boldsymbol{n})$, so that $g(\boldsymbol{n})$ also has zero phase.

Preservation of the Stability: If we begin with a stable IIR prototype filter $p(n)$, the resulting $g(n)$ is also guaranteed to be stable. This is justified as follows: By the definition of bounded-input-bounded-output (BIBO) sta-

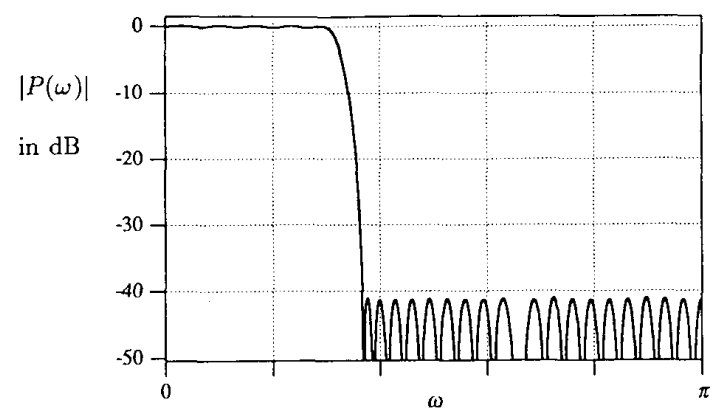

(a)

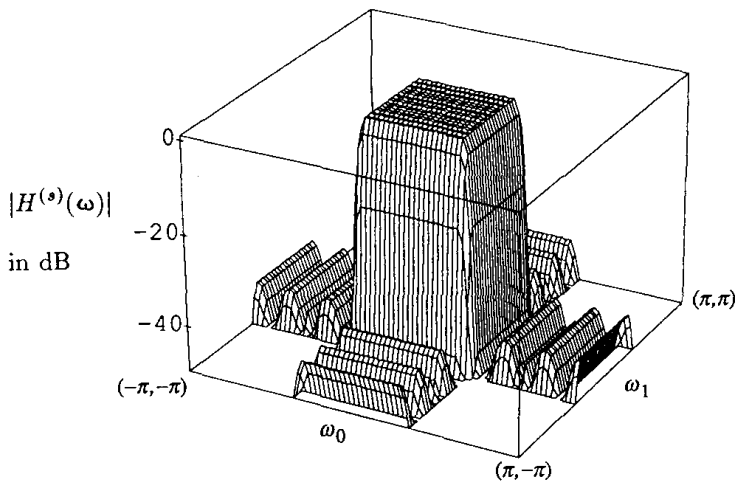

(b)

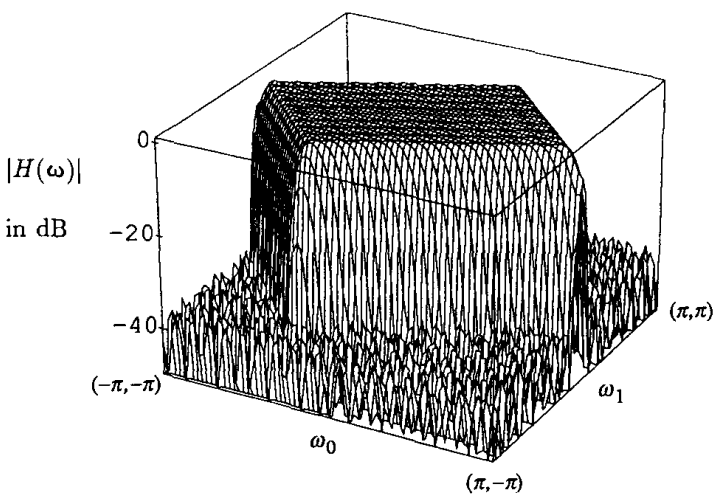

(c)

Fig. 3. Frequency response of filters in design example l: (a) $P(\omega)$, (b) $H^{(1)}(\boldsymbol{\omega}),(\mathrm{c}) H(\mathrm{o})$

bility, $p(n)$ is stable if and only if $\Sigma_{n}|p(n)|$ is finite. Since

$$
\begin{aligned}
\sum_{n}|g(\boldsymbol{n})| & =c \sum_{n}\left|h^{(s)}(\hat{\boldsymbol{M}} \boldsymbol{L n})\right| \leq c \sum_{n}\left|h^{(s)}(\boldsymbol{n})\right| \\
& =c \sum_{n_{0}}\left|p\left(n_{0}\right)\right| \sum_{n_{1}}\left|p\left(n_{1}\right)\right| \cdots \sum_{n D-1}\left|p\left(n_{D-1}\right)\right| \\
& =c\left(\sum_{n}|p(n)|\right)^{D},
\end{aligned}
$$

$\Sigma_{n}|g(n)|$ is also finite. Therefore, $g(\boldsymbol{n})$ is also stable.

Preservation of the Nyquist (Mth Band) Property: A Nyquist filter has impulse response $f(\boldsymbol{n})$ satisfying $f(\boldsymbol{M n})$ $=0$, for all $\boldsymbol{n} \neq \mathbf{0}$, where $\boldsymbol{M}$ is some integer matrix. Such 


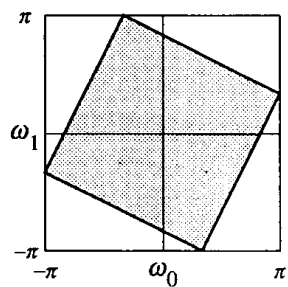

(a)

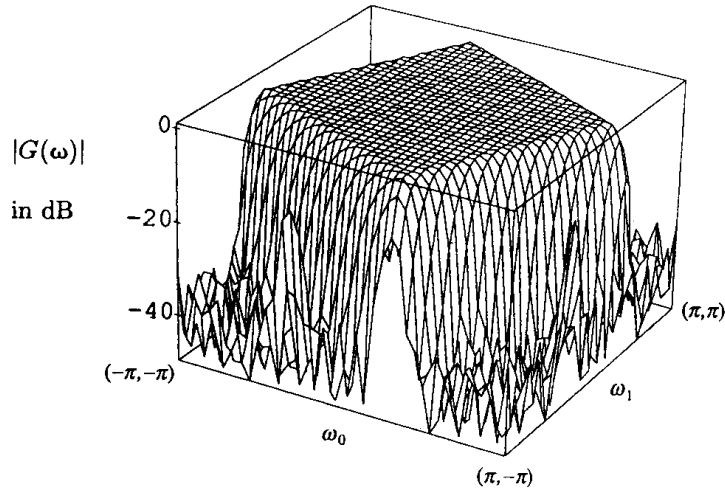

(b)

Fig. 4. The filter $G(\omega)$ in design example 2: (a) desired passband, (b) frequency response.

filters are also called $\boldsymbol{M}$ th band filters. When used as interpolation filters, these filters have the advantage that the values of existing samples can be preserved, i.e., there is no intersymbol interference. With our method, the $\mathrm{Ny}$ quist property is also preserved. More precisely, let $p(n)$ be Nyquist, i.e., $p(J(\boldsymbol{M}) n)=0$, for $n \neq 0$. From (3.1), we therefore have $h^{(s)}(J(\boldsymbol{M}) \boldsymbol{n})=0$, for $\boldsymbol{n} \neq \mathbf{0}$. Then, $h(\boldsymbol{M n})=c_{0} h^{(s)}(\hat{\boldsymbol{M} M n})=c_{0} h^{(s)}(J(\boldsymbol{M}) \boldsymbol{n})=0$, for $\boldsymbol{n} \neq$ $\mathbf{0}$, so $h(\boldsymbol{n})$ is also Nyquist (Mth band). Furthermore, if we generalize the Nyquist property for the rational case to be: $f(\boldsymbol{H n})=\mathbf{0}$ whenever $\boldsymbol{H n}$ is a nonzero integer vector $(\boldsymbol{H}$ th band property), it can be verified that the resulting $g(n)$ has Nyquist property as well.

Separability of the Polyphase Components: When used in multirate applications, e.g., decimation filters, interpolation filters, and maximally decimated filter banks, filters are usually implemented in polyphase components [11], [12]. In these applications, the implementation of a filter is efficient (with complexity $\mathcal{O}(N)$ ) as long as its polyphase components are separable, even though the filter itself may be nonseparable. As shown below, the design procedure mentioned previously results in filters with separable polyphase components. This fact also helps us in designing uniform DFT filter banks, as explained in Section VI later.

Remark: A method of designing 2D diamond-shaped filters having two separable polyphase components was proposed in [3]. From this point of view, our method is indeed a generalization of the results in [3].

Since the polyphase decomposition is defined only with respect to integer matrices, we only have to discuss the filter $h(\boldsymbol{n})$ obtained by using steps 2-4 with respect to the integer matrix $\boldsymbol{M}$. The polyphase components of $h(n)$ with respect to $\boldsymbol{M}$ are defined as [11], [12]

$$
\begin{aligned}
e_{\boldsymbol{k}_{j}}(\boldsymbol{n}) & =h\left(\boldsymbol{M n}+\boldsymbol{k}_{j}\right), & & \text { (Type 1) } \\
\text { or } \quad r_{\boldsymbol{k}_{j}}(\boldsymbol{n}) & =h\left(\boldsymbol{M} \boldsymbol{n}-\boldsymbol{k}_{j}\right), & & \text { (Type 2) }
\end{aligned}
$$

where $\boldsymbol{k}_{j} \in \mathfrak{U}(\boldsymbol{M})$ and $\boldsymbol{k}_{j}$ can take on $J(\boldsymbol{M})$ different values. Usually, $\boldsymbol{k}_{0}$ is taken to be the zero vector $\mathbf{0}$. In the frequency domain, the polyphase decomposition of $H(\boldsymbol{\omega})$ can be expressed as

$$
\begin{aligned}
H(\omega) & =\sum_{\boldsymbol{k}_{j} \in \mathfrak{\Re}(M)} e^{-j \boldsymbol{\omega}^{T} \boldsymbol{k}_{j}} E_{\boldsymbol{k}_{j}}\left(\boldsymbol{M}^{T} \boldsymbol{\omega}\right), & \text { (Type 1) } \\
\text { or } H(\boldsymbol{\omega}) & =\sum_{\boldsymbol{k}_{j} \in \mathfrak{\Re}(M)} e^{j \boldsymbol{\omega}^{T} \boldsymbol{k}_{j}} R_{\boldsymbol{k}_{i}}\left(\boldsymbol{M}^{T} \boldsymbol{\omega}\right) & \text { (Type 2). }
\end{aligned}
$$

Note that the term $e^{-j \omega T_{k}}$ is a delay operator.

We now prove that all these polyphase components are separable. In fact, we can show these polyphase components can be separated into polyphase components of the $1 \mathrm{D}$ filter $P(\omega)$. Consider $e_{k_{i}}(\boldsymbol{n})$, the $j$ th polyphase component of $h(\boldsymbol{n})$. We have

$$
\begin{aligned}
& e_{\boldsymbol{k}_{j}}(\boldsymbol{n})=h\left(\boldsymbol{M n}+\boldsymbol{k}_{j}\right) \\
& =c_{0} h^{(s)}\left(\hat{\boldsymbol{M}} \boldsymbol{M} \boldsymbol{n}+\hat{\boldsymbol{M}} \boldsymbol{k}_{j}\right) \\
& =c_{0} h^{(s)}\left(J(\boldsymbol{M}) \boldsymbol{n}+\hat{\boldsymbol{M}} \boldsymbol{k}_{j}\right) . \\
& \text { Let } \boldsymbol{l}=\left[\begin{array}{llll}
l_{0} & l_{1} & \cdots & l_{D-1}
\end{array}\right]^{T}=\hat{\boldsymbol{M}} \boldsymbol{k}_{j} \text {, then } \\
& e_{\boldsymbol{k}_{j}}(\boldsymbol{n})=c_{0} p\left(J(\boldsymbol{M}) n_{0}+l_{0}\right) p\left(J(\boldsymbol{M}) n_{1}+l_{1}\right) \\
& \cdots p\left(J(\boldsymbol{M}) n_{D-1}+l_{D-1}\right) .
\end{aligned}
$$
be verified that $l \in \mathfrak{N}(J(\boldsymbol{M}) \boldsymbol{I})$, so $0 \leq l_{i} \leq J(\boldsymbol{M})-1$. We thus conclude that $e_{k_{j}}(n)$ 's can be separated into Type 1 polyphase components of $p(n)$.

Causality of Designed Filters: Suppose we start from a ID causal filter $p(n)$. The separable MD filter $h^{(s)}(n)$ obtained in (3.1) is obviously causal (i.e., $h^{(s)}(n)$ is nonzero only when all $n_{i}$ 's are nonnegative). When $h^{(s)}(n)$ is decimated by $\hat{M} \boldsymbol{L}$, the resulting $g(\boldsymbol{n})$ may or may not be causal, depending on $\hat{M} \boldsymbol{L}$. It can be shown that $g(\boldsymbol{n})$ is still causal if and only if all the elements in $(\hat{\boldsymbol{M}} L)^{-1}$ are nonnegative, which is equivalent to the condition that all the elements in $\boldsymbol{H}$ be nonnegative.

Even though $g(\boldsymbol{n})$ may not remain causal for some choices of $\boldsymbol{H}$, this is not a significant problem because we do not implement $g(\boldsymbol{n})$ directly. As shown later in the following section, $h^{(s)}(\boldsymbol{n})$, which is causal and stable as long as $p(n)$ is causal stable, is the filter to be implemented.

\section{EFFICIENT IMPLEMENTATION}

Because of the separability of $H^{(s)}(\omega)$, the proposed method is efficient not only in the design, but also in the implementation. We shall present a polyphase implemen- 
tation in which the complexity (number of arithmetic operations per filter output sample) grows linearly with the filter length $N$, i.e., $\mathcal{O}(N)$, instead of $\mathcal{O}\left(N^{D}\right)$. A similar advantage has been demonstrated for the 2D case in [2].

We shall take the 2D case for our example. Suppose $p(n)$ in step 2 has $N$ coefficients. Therefore, $h^{(n)}(n)$ has $N^{2}$ coefficients and $g(\boldsymbol{n})$ has approximately $N^{2} / J(\hat{\boldsymbol{M}} \boldsymbol{L})$ coefficients. Hence, to implement $g(\boldsymbol{n})$ directly requires approximately $N^{2} / J(\hat{M} L)$ "arithmetic operations per computed output pixel" (OPP's). Instead of direct implementation, we shall derive an efficient implementation as follows.

We can easily verify the decimation equivalence shown in Fig. 5, where $E_{0}(\omega)$ is the Fourier transform of the $\boldsymbol{M}$ fold decimated version of $h(\boldsymbol{n})$. Using this equivalence, we can schematically represent $G(\omega)$ in terms of $H^{(n)}(\omega)$ as in Fig. $7($ a), since $g(\boldsymbol{n})$ is the $\hat{M} L$-fold decimated version of $c h^{(s)}(\boldsymbol{n})$. Because $H^{(*)}(\boldsymbol{\omega})$ is separable, i.e.,

$$
H^{(\omega)}(\omega)=\underbrace{P\left(\omega_{i j}\right)}_{G_{i(1)}(\omega) !} \cdot \underbrace{P\left(\omega_{1}\right)}_{G !(\omega)}
$$

we obtain the implementation in Fig. 7(b). We see this implementation requires only $J(\hat{M} L) \cdot 2 N$ OPP's, where the factor $J(\hat{M} L)$ is due to the $\hat{M} L$-fold expander. Hence we have reduced the complexity from $\mathcal{O}\left(N^{2}\right)$ to $\mathcal{O}(N)$. Note that this holds for the FIR case as well as the IIR case.

Due to the $\hat{M} L$-fold expander, the input to $G_{0}(\omega)$ has several zero-valued samples. Due to the $\hat{M} L$-fold decimator, a large portion of the output of $G_{1}(\omega)$ is dropped. For the FIR case, we can avoid this inefficiency by using polyphase decompositions. We decompose $G_{0}(\boldsymbol{\omega})$ and $G_{1}(\omega)$ into polyphase components with respect to $\hat{M} L$, so

$$
G_{0}(\omega)=\sum_{k_{j} \in \hat{M}\left(\hat{H} L_{)}\right.} e^{j\left(\omega^{\prime} / k_{t}\right.} R_{0, k_{f}}\left((\hat{M} L)^{T} \omega\right), \quad \text { (Type 2) }
$$

and

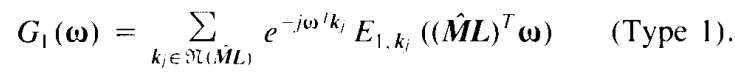

Then, we can use Noble identities (rules which permit us to move decimators and expanders across transfer functions, as shown in Fig. 6) to move the decimators and expanders, and obtain the more efficient implementation in Fig. 7(c), where $s=J(\hat{M} L)$. Now, consider the transfer function, $D_{i, j}(\omega)$, from the output of $R_{0 . k_{i}}(\omega)$ to the input of $E_{1 . k_{i}}(\omega)$, where $\boldsymbol{k}_{i}, \boldsymbol{k}_{j} \in \mathfrak{N}(\hat{M} L)$. Using the decimation equivalence in Fig. 5, we know

$$
D_{i, j}(\boldsymbol{\omega})=\hat{\boldsymbol{M}} \boldsymbol{L} \text {-fold decimated version of } e^{j \omega^{\prime} /\left(\boldsymbol{k}_{i}-\boldsymbol{k}_{j}\right)} .
$$

Then, it can be easily shown that

$$
D_{i, j}(\omega)= \begin{cases}1, & \boldsymbol{k}_{i}=\boldsymbol{k}_{j} \\ 0, & \text { otherwise. }\end{cases}
$$

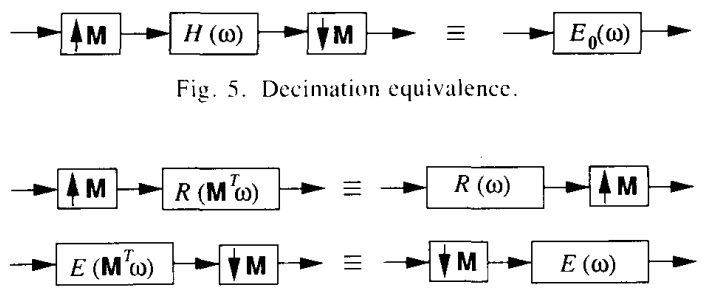

Fig. 6. Noble identities.

Therefore, Fig. 7(c) is equivalent to Fig. 7(d), where the number of required OPP's is reduced to only $2 \mathrm{~N}$.

\section{Remarks:}

1) These discussions hold for FIR as well as IIR cases. For the IIR case, although polyphase technique still works, the implementation we obtain in Fig. 7(d) may require more OPP's than Fig. 7(b).

- 2) Recall that in design example 1, the number of OPP's required for the efficient implementation of Fig. 7(d) is only $2 N=118$, while direct implementation would require approximately $N^{2} / J(\hat{\boldsymbol{M}})=59^{2} / 3 \approx 1160$ OPP's.

3 ) It is easy to extend the above discussion to the MD case. For the MD case, direct implementation of $G(\boldsymbol{\omega})$ requires $N^{D} / J(\hat{\boldsymbol{M}} \boldsymbol{L})$ OPP's. Instead, we can implement it as in Fig. 7(a). Now,

$$
H^{(.)}(\boldsymbol{\omega})=\prod_{i=0}^{D-1} G_{i}(\boldsymbol{\omega})
$$

where $G_{i}(\boldsymbol{\omega})=P\left(\omega_{i}\right)$. We can apply polyphase technique on $G_{0}(\omega)$ and $G_{D-1}(\omega)$, which then requires only $2 N$ OPP's. Since implementing all the other $G_{1}(\omega), \cdots$, $G_{D-2}(\omega)$ still requires $(D-2) J(\hat{M} L) N$ OPP's, the total number of OPP's required is $2 N+(D-2) J(\hat{M} L) N$. The complexity is still in $\mathcal{O}(N)$.

\section{Applications}

Given $\boldsymbol{M}$, we have shown how to design the decimation filter and interpolation filter with respect to $\boldsymbol{M}$. On the other hand, in practical filter design (other than decimation filters and interpolation filters), we are sometimes given the specifications of the passband (the shape of the parallelepiped) instead of $\boldsymbol{M}$. Therefore, we need a systematic way to find the corresponding $\boldsymbol{H}$, so that we can design the filter. To do this, we notice that every parallelepiped-shaped passband can be expressed as

$$
\operatorname{SPD}(\boldsymbol{P})+2 \pi \boldsymbol{m}, \quad \boldsymbol{m} \in \mathfrak{N}
$$

where the columns of $\boldsymbol{P}$ can be called the generating vectors. Fig. 8 shows the generating vectors, $\boldsymbol{p}_{0}$ and $\boldsymbol{p}_{1}$, for a $2 \mathrm{D}$ parallelepiped. Comparing (5.1) with SPD $\left(\pi \boldsymbol{H}^{-T}\right)$, we know that if we let

$$
\boldsymbol{H}=\pi \boldsymbol{P}^{-T}
$$

and follow the design procedure in Section III, the resulting filter will have the desired passband as in (5.1). 

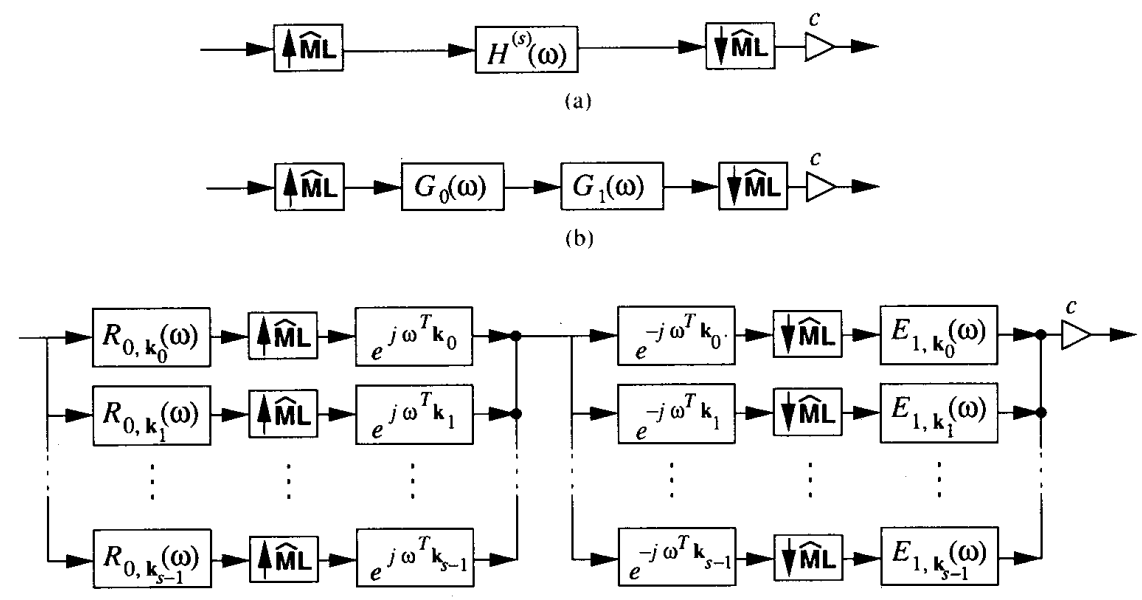

(c)

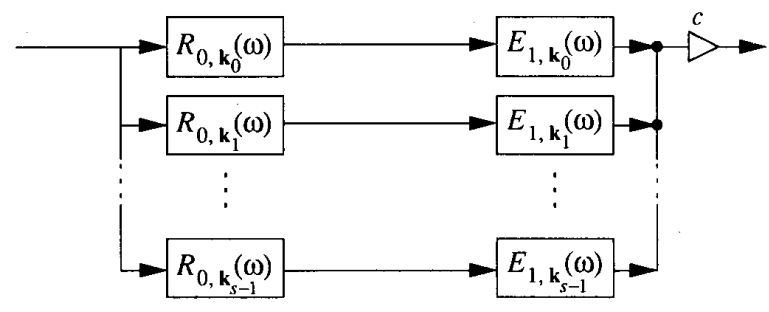

(d)

Fig. 7. Efficient implementation of an MD filter

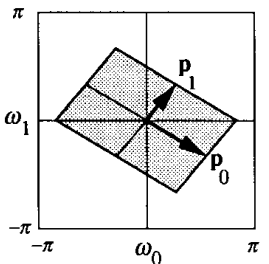

Fig. 8. The generating vectors of a $2 \mathrm{D}$ parallelepiped.

Once we design a filter with some parallelepiped-shaped passband, many other filters can be designed in a straightforward way. Fig. 9 shows some 2D examples of these filters. First, if we choose $p(n)$ in step 2 to be a bandpass filter, we can get $H(\omega)$ as in Fig. 9(a). Second, we know that the modulation in the time domain leads to a shift in the frequency domain, as in the following relation:

$$
h(\boldsymbol{n}) e^{i \boldsymbol{b}^{T} \boldsymbol{n}} \leftrightarrow H(\boldsymbol{\omega}-\boldsymbol{b}) .
$$

Therefore, every filter with a parallelepiped-shaped passband which is not centered at the origin can be obtained simply by modulating a filter with the passband centered at the origin. For example, filters in Fig. 9(b), including the fan filter, can be designed using the proposed approach followed by a proper modulation. Finally, since the sum (or difference) of zero-phase filters is still a zerophase filter with the passband being the sum (or differ- ence) of the passbands of these filters, all the filters in Fig. 9(c), including hexagonal filters, can be obtained easily.

\section{MD UNIFORM DFT QMF BANKS}

The proposed design procedure also applies to the design of MD uniform DFT QMF banks. We shall show that all the properties which can be achieved in 1D uniform DFT QMF banks [18], [19], can be extended into MD using this approach.

Let $J$ denote $J(\boldsymbol{M})$ for simplicity. A $J$-channel maximally-decimated QMF bank is shown in Fig. 10(a), where $\boldsymbol{m}_{i} \in \mathfrak{N}\left(\boldsymbol{M}^{T}\right)$. Notice that $\boldsymbol{m}_{i}$ can take $J$ different values, which is consistent with the number of channels. In general, this system is a linear time-varying (LTV) system. We want to choose analysis filters $H_{m_{i}}(\omega)$ 's and synthesis filters $F_{m_{i}}(\omega)$ 's properly such that this system has the following properties:

1) Passband supports of analysis filters partition the whole frequency range $[-\pi, \pi)^{D}$ and each analysis filter has good frequency response (small ripple sizes).

2) There is no alias distortion (ALD). That is, the system is linear time invariant (LTI), so $\hat{X}(\boldsymbol{\omega})=T(\boldsymbol{\omega}) X(\boldsymbol{\omega})$. We call $T(\omega)$ the overall transfer function of this system.

3) There is no amplitude distortion (AMD). That is, given the system is indeed LTI, we want the overall transfer function $T(\omega)$ to be all pass, i.e., $|T(\omega)|=1$ for all 


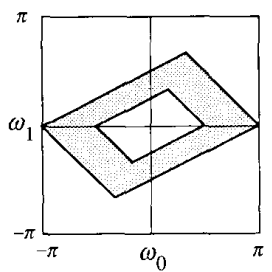

(a)
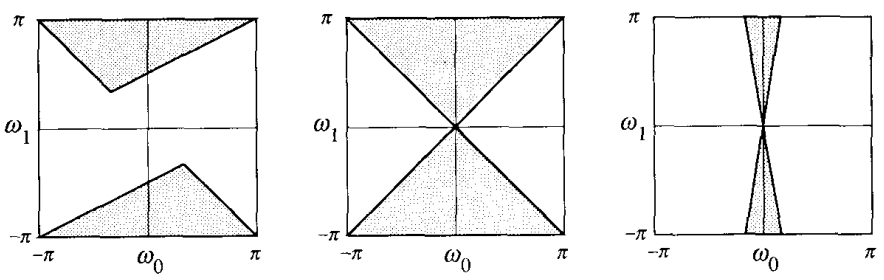

(b)
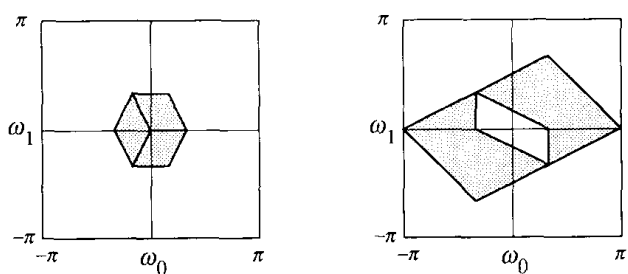

(c)

Fig. 9. Filters which can be obtained by using the proposed method.

$\omega$. When this cannot be satisfied, we want at least the system to have small AMD $(|T(\omega)| \approx 1)$.

4) There is no phase distortion (PHD). That is, given the system is indeed LTI, we want the overall transfer function $T(\omega)$ to have zero phase.

When a system satisfies conditions 2-4, the transfer function $T(\boldsymbol{\omega})$ must be unity so $\hat{x}(\boldsymbol{n})=x(\boldsymbol{n})$ and we say this system achieves perfect reconstruction (PR).

A special QMF bank is the so-called uniform DFT QMF bank, where the analysis filters and the synthesis filters are related as

$$
\begin{aligned}
& H_{m_{i}}(\omega)=H_{m_{i}}\left(\omega-2 \pi M^{-T} m_{i}\right) \\
& F_{m_{i}}(\omega)=F_{m_{0}}\left(\omega-2 \pi M^{-T} m_{i}\right)
\end{aligned}
$$

with $\boldsymbol{m}_{i} \in \mathscr{N}\left(\boldsymbol{M}^{r}\right)$. Because filters in all other channels are completely determined by the filters in the 0th channel, $H_{m_{11}}(\omega)$ and $F_{m_{0}}(\omega)$ are called the prototype analysis filter and synthesis filter, respectively. If we let $H_{m 0}(\omega)$ have passband support in SPD $\left(\boldsymbol{\pi} \boldsymbol{M}^{-T}\right)$, then it can be shown that the passband supports of analysis filters cover the whole frequency range $[-\pi, \pi)^{D}$. It can be verified that this system can be redrawn as Fig. 10(b), where the $E_{k_{i}}(\omega)$ 's are the Type 1 polyphase components of the prototype analysis filter $H_{m 0}(\omega)$ with respect to $M$, and the $R_{k}(\omega)$ 's are the Type 2 polyphase components of the prototype synthesis filter $F_{m i}(\boldsymbol{\omega})$. Also, the $J \times J$ matrix, $W^{(n)}$, is called the generalized DFT matrix [1], [11], with its $(i, j)$ th element defined as

$$
\left[\boldsymbol{W}^{(j)}\right]_{i, j}=e^{-j 2 \pi \boldsymbol{m}_{j} \boldsymbol{M} \cdot \boldsymbol{k}_{i}} \quad \boldsymbol{m}_{i} \in \mathfrak{N}\left(\boldsymbol{M}^{T}\right), \boldsymbol{k}_{j} \in \mathfrak{N}(\boldsymbol{M}) .
$$

Using Noble identities to move the decimators and expanders, we get the equivalent system in Fig. 10(c). It can be shown that $\boldsymbol{W}^{\left.()^{\prime \prime}\right)}$ is unitary, with $\left[\boldsymbol{W}^{()^{\prime \prime}}\right]^{T}\left[\boldsymbol{W}^{(.)}\right]^{*}=\boldsymbol{I}$. Therefore, Fig. 10(c) can be redrawn as Fig. 10(d) (with the scale $J$ omitted).

There are many ways of choosing $E_{k_{i}}(\boldsymbol{\omega})^{\text {'s }}$ and $R_{k_{i}}(\omega)$ 's, depending on properties we want the system to have. For example,

1) We can choose $E_{k_{j}}(\omega)$ 's and $R_{k_{i}}(\omega)$ 's such that

$$
\begin{aligned}
R_{k_{j}}(\omega) E_{k_{i}}(\omega) & =R_{k_{1}}(\omega) E_{k_{1}}(\omega) \\
\text { for } j & =0, \cdots, J-1 .
\end{aligned}
$$

In other words, $R_{k_{j}}(\omega) E_{k_{f}}(\omega)$ is independent with $j$. Then, we get a system that is free from aliasing. For this case, the overall transfer function is

$$
T(\omega)=R_{k+1}\left(M^{T} \omega\right) E_{k 0}\left(M^{T} \omega\right) .
$$

This can be seen by using Noble identities to move all $E_{k_{i}}(\omega)$ 's and $R_{k_{i}}(\omega)$ 's to the right across $\boldsymbol{M}$-fold expanders and realizing that the rest part is an identity system. In fact, (6.3) is necessary and sufficient for the system to be alias-free (see [20] for details). 


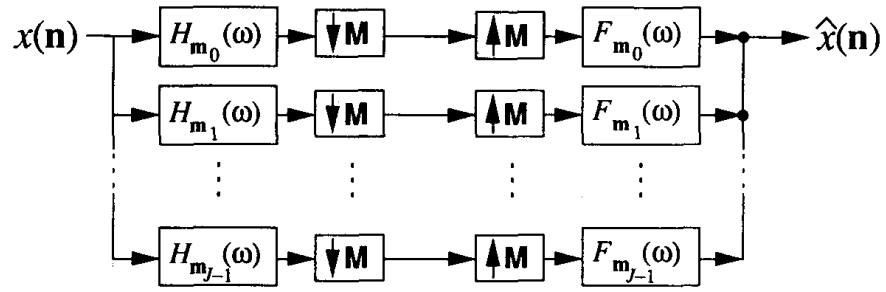

(a)

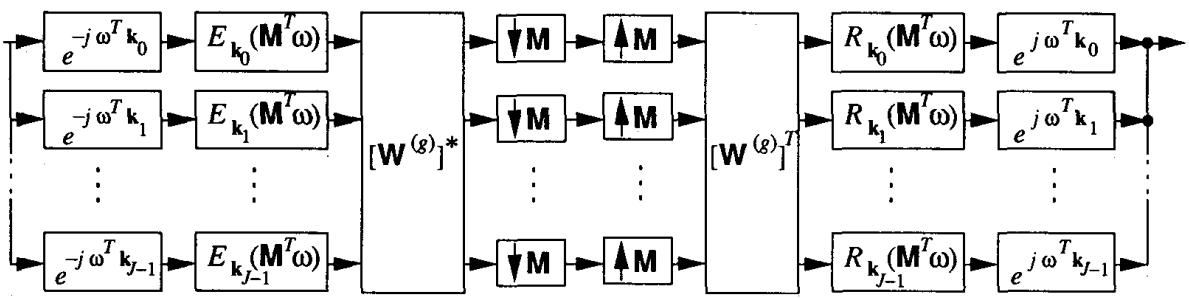

(b)

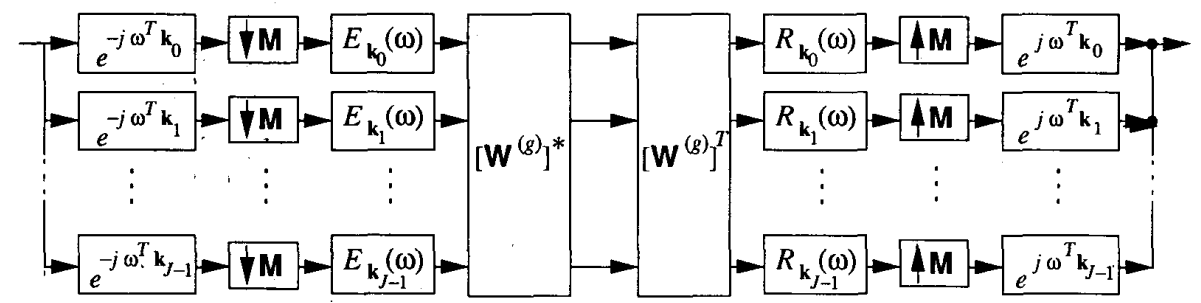

(c)

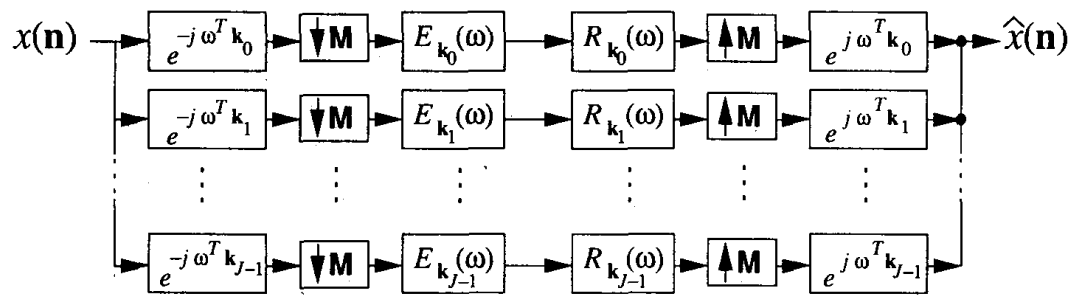

(d)

Fig. 10. MD uniform DFT QMF bank.

2) If we choose $R_{k_{i}}(\omega)=1 / E_{k_{i}}(\omega)$, we get a PR system. However, this choice may result in unstable synthesis filters, just as in the ID case [19].

3) If we choose

$$
R_{\boldsymbol{k}_{i}}(\omega)=\prod_{\substack{\boldsymbol{k}_{i} \in \mathfrak{\Re}(M) \\ i \neq j}} E_{\boldsymbol{k}_{i}}(\omega)
$$

we obtain a system which is free from aliasing and has overall transfer function

$$
T(\boldsymbol{\omega})=\prod_{k_{j} \in \mathfrak{R}(\boldsymbol{M})} E_{k_{j}}\left(\boldsymbol{M}^{T} \boldsymbol{\omega}\right) .
$$

3a) If analysis filters are all FIR, then synthesis filters will also be FIR, hence there is no stability problem. However, for the FIR case, if we want a PR system where $T(\omega)$ equals unity, then all $E_{k_{i}}(\omega)$ 's must be pure delays and hence each analysis filter has only $J$ nonzero coefficients. For nontrivial filters with more than $J$ nonzero coefficients, we can only make $T(\omega)$ zero-phase (no PHD) and optimize coefficients of $E_{k_{i}}(\omega)$ 's (coefficients of $H_{m(0)}(\omega)$ ) such that $|T(\omega)| \approx 1$ (small AMD). The counterpart of this in the $1 \mathrm{D}$ case was presented in [18].

3b) We can choose $E_{k_{j}}(\omega)$ 's to be stable IIR all-pass functions $\left(\left|E_{k_{i}}(\omega)\right|=1\right.$ for all $\left.\omega\right)$. Then, all the analysis filters and synthesis filters as well are stable. From (6.6), we know $|T(\boldsymbol{\omega})|=1$ hence AMD is completely eliminated. In this case, $T(\omega)$ is a stable IIR transfer function so PHD cannot be avoided. In applications where phase information is important (e.g., in 2D im- 
age processing), an all-pass filter can be cascaded with this system to equalize its phase response. The ID counterpart of this can be found in [19], [21].

4) We can choose $E_{\boldsymbol{k}_{i}}(\boldsymbol{\omega})$ 's to be stable IIR all-pass functions (as in Case $3 \mathrm{~b}$ above) and let

$$
R_{k_{i}}(\omega)=E_{k_{i}}^{*}(\omega) \text {. }
$$

Now, in each channel of Fig. 10(d), we have

$$
R_{k_{j}}(\boldsymbol{\omega}) E_{k_{j}}(\boldsymbol{\omega})=E_{k_{i}}^{*}(\boldsymbol{\omega}) E_{k_{j}}(\boldsymbol{\omega})=\left|E_{k_{j}}(\boldsymbol{\omega})\right|^{2}=1 .
$$

So, $T(\omega)=1$ and we get a PR system. Note that $(6.7)$ implies $r_{k_{i}}(\boldsymbol{n})=e_{k_{i}}^{*}(-\boldsymbol{n})$ so $r_{k_{i}}(\boldsymbol{n})$ is also stable. However. if $e_{k}(n)$ are chosen to be causal, $r_{k_{i}}(\boldsymbol{n})$ becomes anticausal. This problem of anticausal synthesis filters can be solved by running the filters backwards [3], [22], [23].

Although the above theoretical derivation may seem to be easy, direct optimization of filter coefficients is often unreasonably difficult due to the large number of parameters to be optimized, especially when $D$ is large. Furthermore, the large number of OPP's required in direct implementation is also a problem. Our approach overcomes these two difficulties. We now show that starting from an appropriate ID uniform DFT QMF bank, we can derive an MD uniform DFT QMF bank with $H_{m(1}(\omega)$ having support $\operatorname{SPD}\left(\pi \boldsymbol{M}^{-T}\right)$.

\section{Design Procedure}

Step 1. Design the prototype analysis/synthesis filters, $P_{0}(\omega)$ and $Q_{0}(\omega)$, of a ID $J$-channel uniform DFT QMF bank. Let $P_{0}(\omega)$ have passband support in $[\pi / J, \pi / J)$.

Step 2. Construct the separable MD filters $H_{k+1}^{(s)}(\boldsymbol{\omega})$ and $F_{k i}^{(s)}(\boldsymbol{\omega})$ as

$$
\begin{aligned}
& H_{k \mid 1}^{(s)}(\omega)=P_{0}\left(\omega_{0}\right) P_{0}\left(\omega_{1}\right) \cdots P_{0}\left(\omega_{D-1}\right) \\
& F_{k_{1}}^{(s)}(\omega)=Q_{0}\left(\omega_{0}\right) Q_{0}\left(\omega_{1}\right) \cdots Q_{0}\left(\omega_{D-1}\right) .
\end{aligned}
$$

Step 3. Define $h_{\boldsymbol{m}(0)}(\boldsymbol{n})=h_{k_{10}}^{(s)}(\hat{\boldsymbol{M}} \boldsymbol{n})$ and $f_{\boldsymbol{m} \mid}(\boldsymbol{n})=$ $f_{k_{11}}^{(s)}(\hat{\boldsymbol{M}} \boldsymbol{n})$. That is, decimate $h_{k_{1}}^{(s)}(\boldsymbol{n})$ and $f_{k_{1}}^{(n)}(\boldsymbol{n})$ by $\hat{\boldsymbol{M}}$. As explained previously, the resulting $H_{m,}(\omega)$ has support in $\operatorname{SPD}\left(\pi \boldsymbol{M}^{-T}\right)$.

After $H_{m_{0}}(\omega)$ and $F_{m_{0}}(\omega)$ are obtained, we may implement the MD QMF bank as in Fig. 10(c). It has been shown in Section III that all the $E_{k_{i}}(\boldsymbol{\omega})$ 's and $R_{k_{i}}(\boldsymbol{\omega})$ 's are separable, even though $H_{m_{i}}(\boldsymbol{\omega})$ and $F_{m_{0}}(\boldsymbol{\omega})$ are not separable. Therefore, this indeed gives a very efficient implementation.

Since filters in an MD DFT filter bank are all determined by one prototype filter, it is not counter-intuitive that we can apply our method in Section III to the prototype filter of a ID DFT filter bank and obtain the MD prototype filter. However, the following claim is nontrivial:

Claim: The $H_{m_{0}}(\boldsymbol{\omega})$ and $F_{m_{01}}(\boldsymbol{\omega})$ obtained are the desired prototype filters for a $J$-channel MD uniform DFT QMF bank. This filter bank will have all the properties that the original 1D $J$-channel uniform DFT QMF bank has. For example, if the 1D DFT filter bank system is PR. the resulting MD DFT filter bank system is also PR. Similarly, the design method preserves other properties like: no ALD, no or small AMD, and no PHD.

\section{Justification}

Consider the $J$-channel ID uniform DFT QMF bank which we start from. Let $B_{k}(\omega)$ 's denote Type 1 polyphase components of the prototype analysis filter $P_{0}(\omega)$, and $S_{k}(\omega)$ 's denote Type 2 polyphase components of the prototype synthesis filter $Q_{0}(\omega)$. Suppose this is a system without aliasing, i.e.,

$$
\begin{aligned}
& S_{k}(\omega) B_{k}(\omega)=S_{0}(\omega) B_{0}(\omega) \\
& \qquad \text { for } k=0, \cdots, J-1 .
\end{aligned}
$$

It has been proved in Section III that polyphase components of the resulting MD filters are related with those of 1D filters as

$$
E_{k i}(\omega)=B_{l_{1}}\left(\omega_{0}\right) \cdots B_{l b, 1}\left(\omega_{D-1}\right)
$$

and

$$
R_{k_{i}}(\omega)=S_{l 0}\left(\omega_{0}\right) \cdots S_{l D}\left(\omega_{D-1}\right)
$$

where $\left[\begin{array}{llll}l_{0} & l_{1} & \cdots & l_{D-1}\end{array}\right]^{T}=\boldsymbol{l}=\hat{\boldsymbol{M}} \boldsymbol{k}_{j}$. Considering $R_{k_{i}}(\omega) E_{k_{i}}(\omega)$, we obtain

$$
\begin{aligned}
R_{k_{j}}(\omega) E_{k_{i}}(\omega) & =\prod_{i=0}^{D-1} S_{l_{i}}\left(\omega_{i}\right) B_{l_{i}}\left(\omega_{i}\right) \\
& =\prod_{i=0}^{D-1} S_{0}\left(\omega_{i}\right) B_{0}\left(\omega_{i}\right) .
\end{aligned}
$$

Since $R_{k_{j}}(\omega) E_{k_{i}}(\boldsymbol{\omega})$ is independent with $j$, the resulting MD QMF bank is also free from aliasing with overall transfer function

$$
T(\boldsymbol{\omega})=R_{\boldsymbol{k}_{11}}\left(\boldsymbol{M}^{T} \boldsymbol{\omega}\right) E_{k_{11}}\left(\boldsymbol{M}^{T} \boldsymbol{\omega}\right) .
$$

Given that both the 1D and MD DFT QMF banks are alias free, let us take a closer look at these transfer functions. Define $\boldsymbol{e}_{i}$ to be a vector with zero elements except that the $i$ th element is unity. Then, (6.12) can be written as

$$
R_{k_{j}}(\boldsymbol{\omega}) E_{k_{j}}(\boldsymbol{\omega})=\prod_{i=0}^{D-1} S_{0}\left(\boldsymbol{e}_{i}^{T} \boldsymbol{\omega}\right) B_{0}\left(\boldsymbol{e}_{i}^{T} \boldsymbol{\omega}\right)
$$

and (6.13) becomes

$$
T(\boldsymbol{\omega})=\prod_{i=0}^{D-1} S_{0}\left(\boldsymbol{e}_{i}^{T} \boldsymbol{M}^{T} \boldsymbol{\omega}\right) B_{0}\left(\boldsymbol{e}_{i}^{T} \boldsymbol{M}^{T} \boldsymbol{\omega}\right)
$$

Let $V(\omega)$ denote the overall transfer function of the $1 \mathrm{D}$ QMF bank. Under the alias-free condition, we know $V(\omega)$ $=B_{0}(J \omega) S_{0}(J \omega)$. Define $T^{(1)}(\omega)$ as

$$
T^{(s)}(\omega)=\prod_{i=0}^{D-1} V\left(\omega_{i}\right) .
$$


Since $J \omega_{i}=J e_{i}^{T} \omega=e_{i}^{T} M^{T} \hat{M}^{T} \omega$, (6.16) can be written as

$$
\begin{aligned}
T^{(s)}(\omega) & =\prod_{i=0}^{D-1} S_{0}\left(J \omega_{i}\right) B_{0}\left(J \omega_{i}\right) \\
& =\prod_{i=0}^{D-1} S_{0}\left(\boldsymbol{e}_{i}^{T} \boldsymbol{M}^{T} \hat{\boldsymbol{M}}^{T} \boldsymbol{\omega}\right) B_{0}\left(\boldsymbol{e}_{i}^{T} \boldsymbol{M}^{T} \hat{\boldsymbol{M}}^{T} \boldsymbol{\omega}\right) .
\end{aligned}
$$

Comparing (6.15) and (6.17), we see that

$$
T^{(s)}(\boldsymbol{\omega})=T\left(\hat{\boldsymbol{M}}^{T} \boldsymbol{\omega}\right)
$$

i.e., $T^{(s)}(\omega)$ is exactly the $\hat{\boldsymbol{M}}$-fold expanded version of $T(\omega)$. Note that $(6.18)$ can be written as

$$
T(\omega)=T^{(s)}\left(\hat{M}^{-T} \omega\right) \text {. }
$$

Also note that $T(\omega)$ is the $\hat{M}$-fold decimated version of $T^{(s)}(\omega)$ so that the transfer function of the designed MD QMF bank can be obtained by $\hat{\boldsymbol{M}}$-fold decimating the product of the $1 \mathrm{D}$ overall transfer function $V(\omega)$ in each dimension.

Now, it is easy to show that other properties about AMD, PHD, PR are preserved.

1) Given there is no ALD, we need to discuss the AMD properties.

1a) For the FIR case, we can optimize filter coefficients in the 1D filter bank such that $|V(\omega)| \approx 1 \forall \omega$. From (6.16) and (6.19), we see that $|T(\omega)| \approx 1 \forall \omega$, too. That is, AMD remains small. More specifically, if $V(\omega)$ has ripple size $\delta$, the resulting ripple sizes of both $T^{(s)}(\omega)$ and $T(\omega)$ are at most $D \delta$.

1b) For the IIR case, we can start from ID prototype filters with all-pass polyphase components and make $V(\omega)$ to be all pass (no AMD). From (6.16) and (6.19), we can see that $T(\boldsymbol{\omega})$ is still an all-pass function. Therefore, there is no AMD in the resulting MD filter bank. 2) Given there is no ALD, suppose the $1 \mathrm{D}$ overall transfer function $V(\omega)$ has zero phase, i.e., $V(\omega)$ is real for all $\omega$. From (6.16) and (6.19), we can conclude that $T(\omega)$ is real for all $\omega$ (zero phase) so there is no PHD.

3) Finally, suppose the original system satisfies PR so that $V(\omega)=1$. From (6.16) and (6.19), we can easily conclude that $T(\boldsymbol{\omega})=1$ so $\mathrm{PR}$ is preserved.

There are many useful 1D DFT filter banks in literature [18], [19], [21], [22]. We can apply our method to each of these and derive many useful MD filter banks. In the following, we present several examples.

\section{A. MD Filter Bank Derived from Johnston's Filters (Two-Channel Case)}

Design Example 3: The most commonly used 1D DFT QMF bank is the two-channel FIR case. Johnston designed such filter banks for various specifications and the optimized filter coefficients were tabulated [24], [25]. These systems have no ALD, no PHD, and small AMD. Fig. 11(a) shows the frequency response of the prototype analysis filter $P_{0}(\omega)$ (which is named 32D in Johnston's table). We can apply our method to derive a two-channel

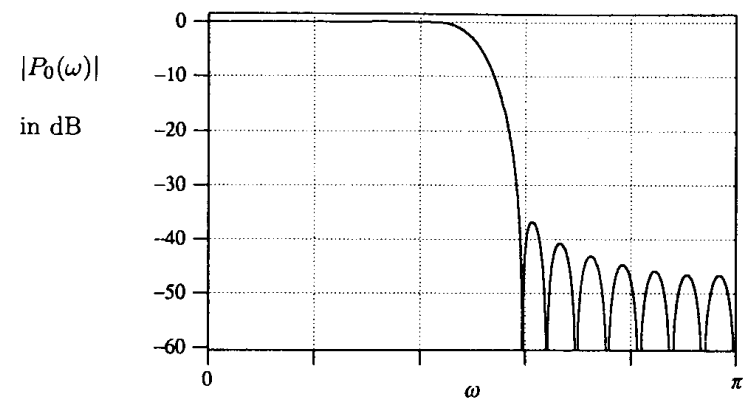

(a)
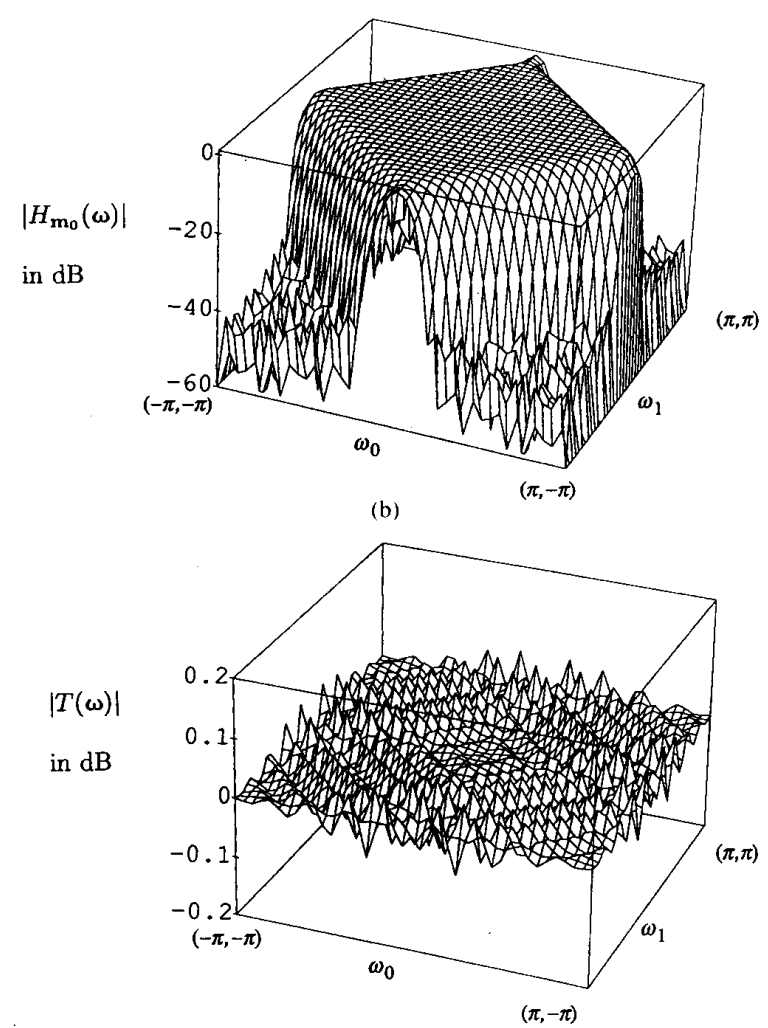

(c)

Fig. 11. Frequency response of filters in design example 3: (a) $P_{0)}(\omega)$, (b) $H_{m},(\omega)$, (c) $T(\omega)$.

MD DFT QMF bank for any decimation matrix $\boldsymbol{M}$ with $J(\boldsymbol{M})=2$. As an example, we choose

$$
\boldsymbol{M}=\left[\begin{array}{rr}
1 & 1 \\
-1 & 1
\end{array}\right]
$$

which defines the so-called quincunx lattice. Applying the design procedure, we obtain the $2 \mathrm{D}$ prototype analysis filter $H_{m 0}(\omega)$, as shown in Fig. 11(b). According to relations in $(6.1)$, the other analysis filter $H_{m_{1}}(\omega)$ is obtained by shifting $H_{m_{0}}(\boldsymbol{\omega})$, i.e., $H_{m_{1}}\left(\omega_{0}, \omega_{1}\right)=H_{m_{0}}\left(\omega_{0}-\pi, \omega_{1}\right.$ $-\pi$ ). The overall transfer function $T(\omega)$, which is zerophase and has magnitude close to unity, is shown in Fig. $11(\mathrm{c})$. 

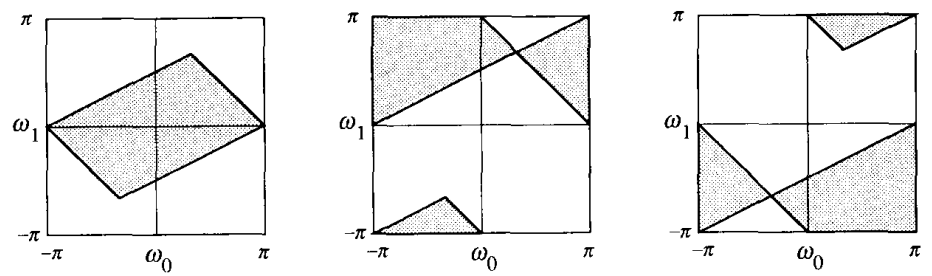

Fig. 12. Passband supports of analysis filters in design example 4.

Remark: The idea of using Johnston's results to derive 2D quincunx QMF bank has been proposed in [26]. Our method generalizes this result and is able to derive any two-channel DFT QMF bank for arbitrary $\boldsymbol{M}$ (with $J(\boldsymbol{M})$ $=2$ ) and any number of dimensions.

\section{B. MD FIR Filter Bank (with More than Two Channels)}

Design Example 4: This is also an FIR example. Let $\boldsymbol{M}$ be the same as in design example 1, so there are three channels and the passband supports of these three analysis filters can be chosen as in Fig. 12. We use the coefficients obtained in example 2 of [18] to form $P_{0}(\omega)$, the optimal zero-phase prototype analysis filter of a 3-channel 1D uniform DFT QMF bank. $P_{0}(\omega)$ has length $N=49$, passband ripple $\delta_{1}=0.001406$, and stopband ripple $\delta_{2}=$ $0.003245(-49.78 \mathrm{~dB})$, as shown in Fig. 13(a). For this ID filter bank, the overall transfer function is close to unity with the ripple size $\delta=0.004226$. Following the steps described earlier, we obtain $H_{m, 1}(\omega)$, which is shown in Fig. 13(b), with passband ripple $\delta_{1}^{\prime}=0.003398$, and stopband ripple $\delta_{2}^{\prime}=0.00570(-44.88 \mathrm{~dB})$. The resulting overall transfer function $T(\omega)$ is also shown in Fig. 13(c), which has ripple size $\delta^{\prime}=0.00847$.

\section{MD IIR All-Pass-Based Filter Bank}

Design Example 5: We now present an example with IIR filters. We start from a ID DFT QMF bank in which the prototype analysis filter $P_{0}(\omega)$ has all-pass polyphase components. For the ID two channel case, is has been described how the design of common digital IIR filters can be modified to design IIR filters with two all-pass polyphase components and how this can be used in twochannel QMF banks [21]. Such a system gives very efficient implementation of alias-free filter banks with no AMD. For example, by using only one multiplication per input sample in the analysis filter bank, we can obtained analysis filters with more than $37-\mathrm{dB}$ stopband attenuation, as illustrated later. For the case of more channels, the design of ID IIR filters with all-pass polyphase components has also been addressed in [27]. Our method can be applied to derive MD DFT QMF banks from results in both [21] and [27]. Again, let us consider the quincunx case for simplicity. We start from a ID fifth-order prototype filter $P_{0}(z)$ with two all-pass polyphase components. More specifically, let $P_{0}(z)=E_{0}\left(z^{2}\right)+z^{-1} E_{1}\left(z^{2}\right)$

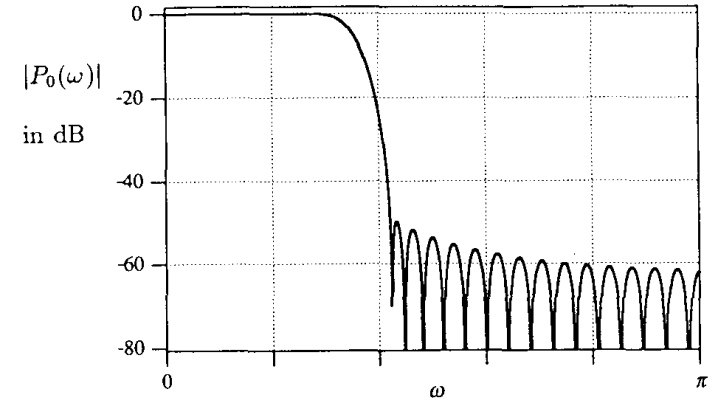

(a)

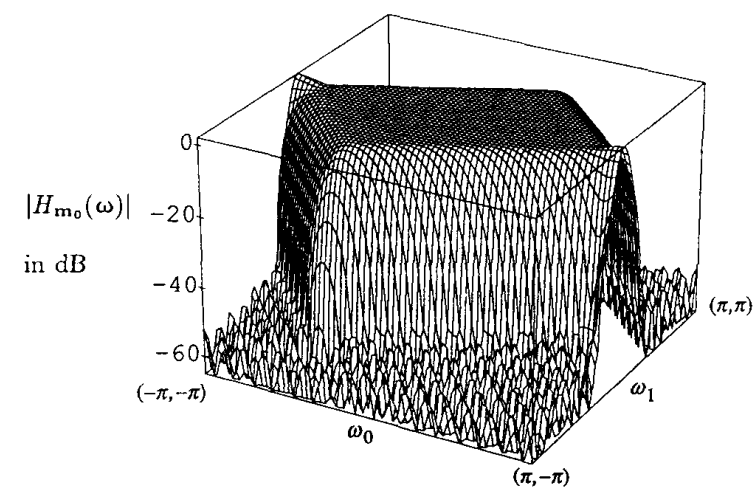

(b)

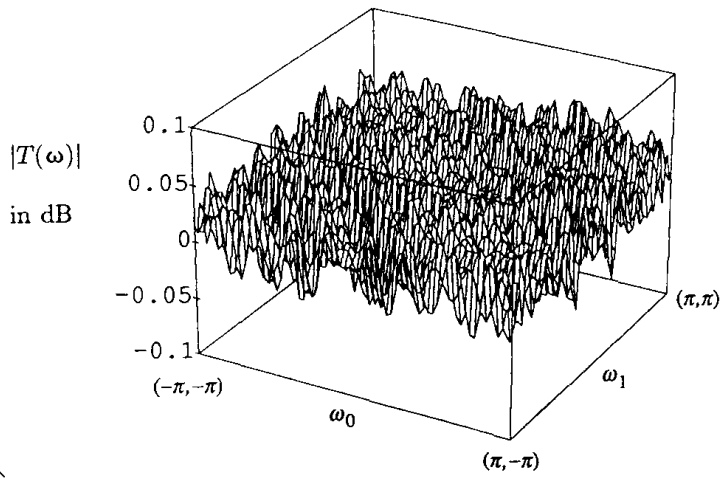

(c)

Fig. 13. Frequency response of tilters in design example 4: (a) $P_{11}(\omega)$. (b) $H_{m, \mathrm{v}}(\boldsymbol{w}),(\mathrm{c}) T(\boldsymbol{w})$ 


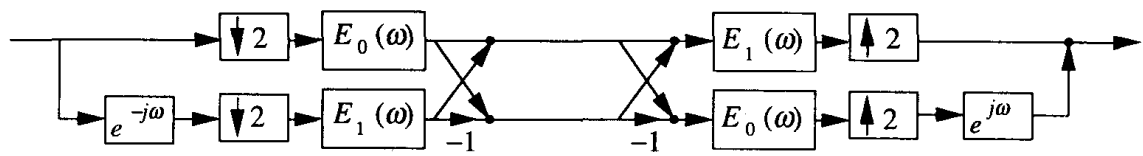

Fig. 14. One-dimensional IIR QMF bank with no AMD

where

$$
\begin{aligned}
& E_{0}(z)=0.5 \frac{\alpha_{0}+z^{-1}}{1+\alpha_{0} z^{-1}} \quad \text { and } \\
& E_{1}(z)=0.5 \frac{\alpha_{1}+z^{-1}}{1+\alpha_{1} z^{-1}} .
\end{aligned}
$$

If we choose the synthesis filters as in Fig. 14, the overall transfer function of this $1 \mathrm{D}$ QMF bank is $2 E_{0}\left(z^{2}\right) E_{1}\left(z^{2}\right)$. Using the method described in [21], we obtain $\alpha_{0}=$ 0.226634 and $\alpha_{1}=0.703653$. The frequency response of $P_{0}(\omega)$ is shown in Fig. 15(a). Applying our method, we can obtain the MD prototype analysis filter $H_{m_{0}}$ with allpass and separable polyphase components (as proved in Section III)

$$
\begin{aligned}
& E_{k_{0}}(\omega)=E_{0}\left(\omega_{0}\right) E_{0}\left(\omega_{1}\right) \quad \text { and } \\
& E_{k_{i}}(\omega)=E_{1}\left(\omega_{0}\right) E_{1}\left(\omega_{1}\right) .
\end{aligned}
$$

The frequency response of $H_{m_{0}}(\omega)$ is shown in Fig. 15(b). Then, we can choose the polyphase component of synthesis filters as in (6.5) and obtain the system in Fig. 16 which is free from ALD and AMD. This system has the overall transfer function $T(\omega)=2 E_{k_{10}}\left(M^{T} \boldsymbol{\omega}\right) E_{k_{1}}\left(M^{T} \omega\right)$ which is indeed all pass. The phase response of $T(\omega)$ is shown in Fig. 15(c), which is not zero phase and hence PHD exists. When PHD is not desired, we can cascade the system with an all-pass function to equalize the phase response. The overall group delays $\tau_{0}(\omega)$ and $\tau_{1}(\omega)$, which are defined as

$$
\begin{aligned}
& \tau_{0}(\omega) \triangleq-\frac{\partial}{\partial \omega_{0}}[\text { phase of } T(\omega)] \quad \text { and } \\
& \tau_{0}(\omega) \triangleq-\frac{\partial}{\partial \omega_{1}}[\text { phase of } T(\omega)]
\end{aligned}
$$

are shown in Figs. 17(a) and (b). Note that all 2D filters in this case are separable and only four multipliers are required in analysis filters. Furthermore, since these multipliers operate at half of the input rate, we need only two multiplications per input pixel to obtain analysis filters with more than 35-dB stopband attenuation.

Remark: Although we use the 2D quincunx case as the example, the proposed method applies to arbitrary $\boldsymbol{M}$ and arbitrary number of dimensions.

\section{MD IIR Perfect Reconstruction Filter Bank}

Design Example 6: We can choose analysis filters same as in design example 5 , but we choose synthesis filters according to (6.7) instead. Hence, we obtain the system

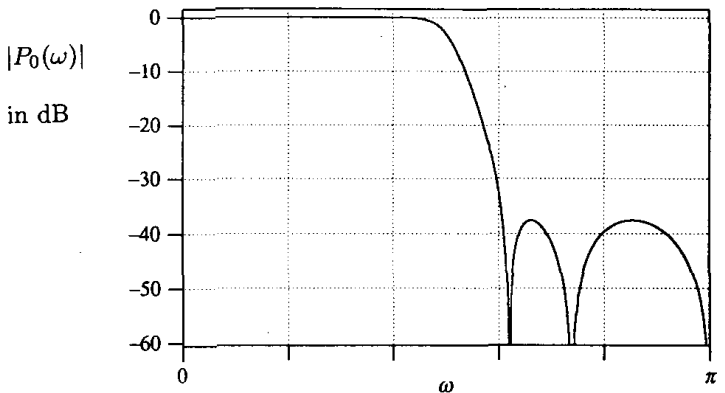

(a)

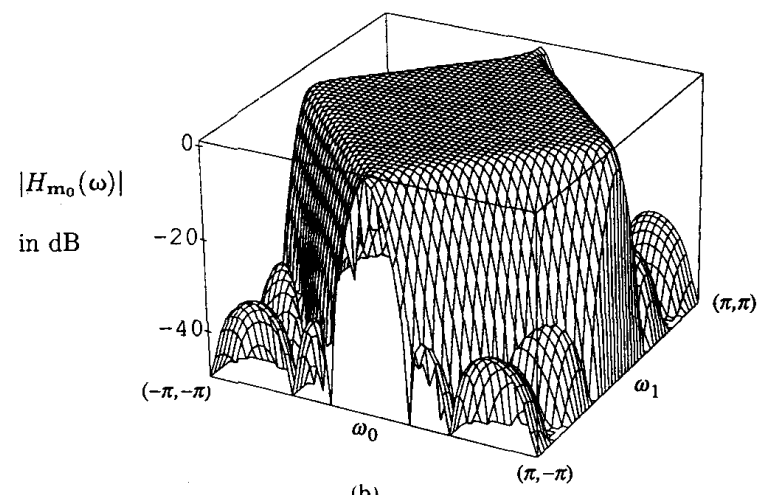

(b)

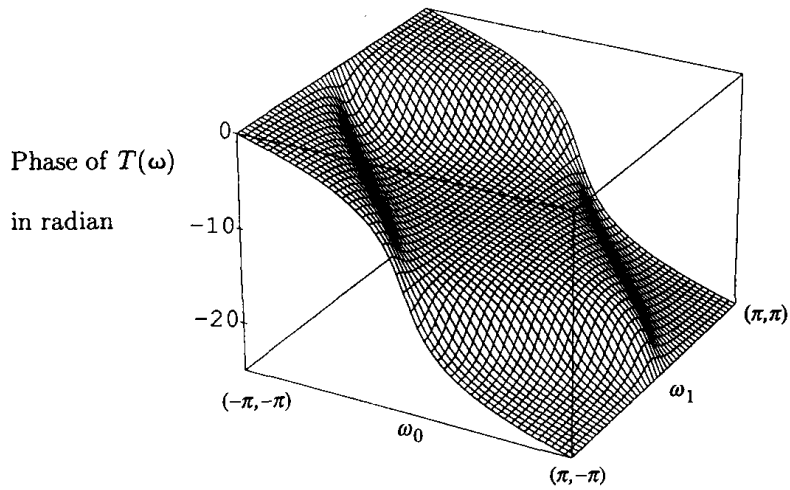

(c)

Fig. 15. Frequency response of filters in design example 5: (a) $P_{0}(\omega)$, (b) $H_{m i}(\omega)$. (c) $T(\omega)$.

in Fig. 18 which achieves PR. For the quincunx case, this idea has been presented in [3]. Our method generalizes this to arbitrary $\boldsymbol{M}$ and arbitrary number of dimensions. Note that synthesis filters in this case are stable but anticausal. This can be solved by running the filters backwards [22] with properly chosen initial conditions [23]. 


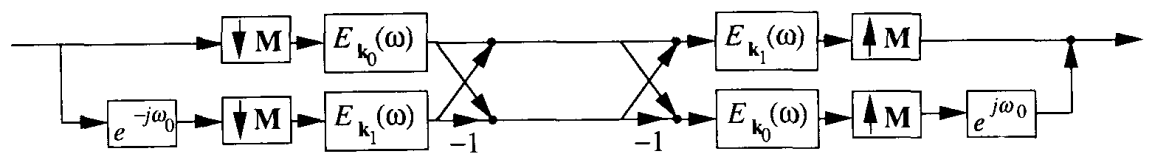

Fig. 16. Polyphase implementation of an MD IIR QMF bank.

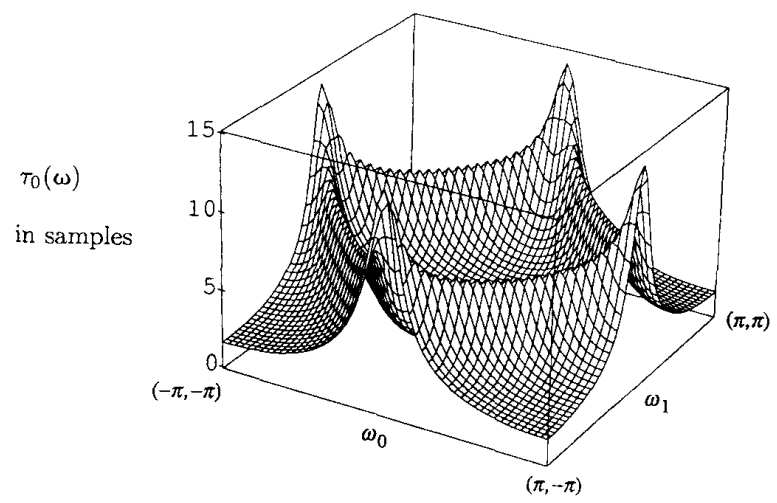

(a)

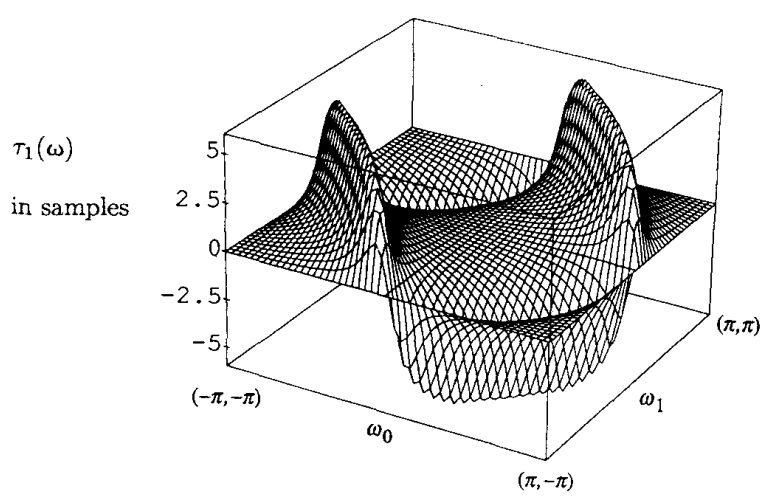

(b)

Fig. 17. Group delays of design example $5:$ (a) $\tau_{11}(\omega)$, (b) $\tau_{1}(\omega)$.

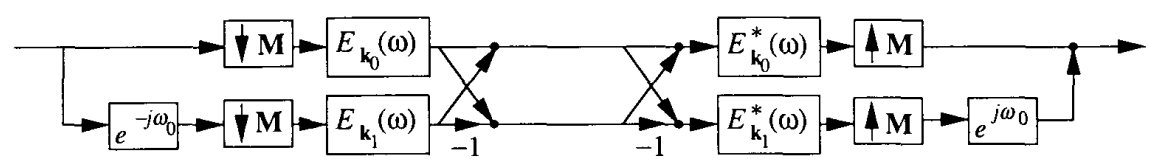

Fig. 18. MD IIR QMF bank with PR property (design example 6).

In summary, since our method results in MD filters with separable polyphase components, all the results in [22] and [23] can be extended to the MD case automatically.

\section{Modification of the Design Procedure}

The value of $J(\boldsymbol{M})$ may be very large in some applications, so the passband of the 1D prototype $P(\omega)$ becomes very narrow. In this case, the required filter order is very large. To deal with this problem, one can use interpolated FIR (IFIR) approach [28] to design $P(\omega)$. The other way is to exploit the common factors (if not unity) in each row of $\boldsymbol{M}$, as follows:
Step 1. Decompose $\boldsymbol{M}$ into $\boldsymbol{M}=\boldsymbol{M}_{p} \boldsymbol{\Lambda}$, where $\boldsymbol{\Lambda}$ is a diagonal matrix with diagonal elements $\lambda_{i}>0$, and $\lambda_{i}$ is the greatest common divisor of the elements in the $i$ th column of $\boldsymbol{M}$.

Step 2. Design the 1D low-pass filters $p_{0}(n), \cdots$, $p_{D-1}(n)$. Each filter $p_{i}(n)$ should have the passband region

$$
\left\lceil-\frac{\pi}{J\left(M_{p}\right) \lambda_{i}} \cdot \frac{\pi}{J\left(M_{p}\right) \lambda_{i}}\right) .
$$

It is easy to see that all these filters have passband wider than $[-\pi / J(\boldsymbol{M}), \pi / J(\boldsymbol{M}))$, thus requiring less filter order than in Section III. 
Step 3. Construct $h^{(s)}(\boldsymbol{n})$ as

$$
h^{(s)}(\boldsymbol{n})=p_{0}\left(n_{0}\right) p_{1}\left(n_{1}\right) \cdots p_{D-1}\left(n_{D-1}\right),
$$

so

$$
H^{(s)}(\omega)=P_{0}\left(\omega_{0}\right) P_{1}\left(\omega_{1}\right) \cdots P_{D-1}\left(\omega_{D-1}\right) .
$$

Step 4. Define $h(n) \triangleq c_{0} h^{(s)}\left(\hat{M}_{p} n\right)$, where $\hat{\boldsymbol{M}}_{p} \triangleq$ $J\left(\hat{\boldsymbol{M}}_{p}\right) \cdot \hat{\boldsymbol{M}}_{\boldsymbol{p}}^{-1}$ and $c_{0}=J\left(\hat{\boldsymbol{M}}_{\boldsymbol{p}}\right)$.

We have to show that $H(\omega)$ has the desired passband. From $(7.2), H^{(s)}(\omega)$ has passband

$$
\begin{aligned}
\frac{\pi}{J\left(M_{p}\right)} \Lambda^{-1} x+2 \pi k^{\prime}, \\
\quad x \in[-1,1)^{D}, \quad \boldsymbol{k}^{\prime} \in \mathscr{N} .
\end{aligned}
$$

From (2.1), with $\boldsymbol{M}$ replaced by $\hat{\boldsymbol{M}}_{p}$, we know that $H(\omega)$ then has passband

$$
\begin{aligned}
& \hat{\boldsymbol{M}}_{p}^{T}\left(\frac{\pi}{J\left(\boldsymbol{M}_{p}\right)} \boldsymbol{\Lambda}^{-1} \boldsymbol{x}+2 \pi \boldsymbol{k}^{\prime}\right)+2 \pi \boldsymbol{k} \\
& \quad \boldsymbol{x} \in[-1,1)^{D}, \quad \boldsymbol{k}^{\prime} \in \mathfrak{K}, \boldsymbol{k} \in \mathfrak{K}\left(\hat{\boldsymbol{M}}_{p}^{T}\right) \\
= & \frac{\pi}{J\left(\boldsymbol{M}_{p}\right)} \hat{\boldsymbol{M}}_{p}^{T} \boldsymbol{\Lambda}^{-1} \boldsymbol{x}+2 \pi \hat{\boldsymbol{M}}_{p}^{T} \boldsymbol{k}^{\prime}+2 \pi \boldsymbol{k} \\
= & \pi \boldsymbol{M}_{p}^{-T} \boldsymbol{\Lambda}^{-1} \boldsymbol{x}+2 \pi\left(\hat{\boldsymbol{M}}_{p}^{T} \boldsymbol{k}^{\prime}+\boldsymbol{k}\right) \\
= & \pi \boldsymbol{M}^{-T} \boldsymbol{x}+2 \pi \boldsymbol{x} \quad \boldsymbol{x} \in[-1,1)^{D}, \boldsymbol{m} \in \mathfrak{N} .
\end{aligned}
$$

Now, we will show that Nyquist and zero-phase properties are still preserved by the modified method.

Proof: Suppose that all $p_{i}(n)$ 's are Nyquist, i.e., $p_{i}\left(J\left(\boldsymbol{M}_{p}\right) \lambda_{i} n\right)=0$ for $n \neq 0$. From (7.1), we therefore have $h^{(*)}\left(J\left(\boldsymbol{M}_{p}\right) \mathbf{\Lambda} \boldsymbol{n}\right)=0$, for $\boldsymbol{n} \neq \mathbf{0}$. So,

$$
\begin{aligned}
h(\boldsymbol{M n}) & =c_{0} h^{(s)}\left(\hat{\boldsymbol{M}}_{\boldsymbol{p}} \boldsymbol{M n}\right)=c_{0} h^{(s)}\left(J\left(\boldsymbol{M}_{\boldsymbol{p}}\right) \boldsymbol{M}_{\boldsymbol{p}}^{-1} \boldsymbol{M} \boldsymbol{n}\right) \\
& =c_{0} h^{(s)}\left(J\left(\boldsymbol{M}_{\boldsymbol{p}}\right) \boldsymbol{\Lambda} \boldsymbol{n}\right)=0, \quad \text { for } \boldsymbol{n} \neq \mathbf{0} .
\end{aligned}
$$

Therefore, $H(\omega)$ is also Nyquist ( $M$ th band). Next, suppose that all $p_{i}(n)$ 's have zero phase, i.e., $p_{i}(n)=$ $p_{i}^{*}(-n), \forall i$. From $(7.1)$, we have $h^{(s)}(\boldsymbol{n})=h^{(*))^{*}}(-\boldsymbol{n})$. Then,

$$
h(\boldsymbol{n})=c_{0} h^{(s)}\left(\hat{\boldsymbol{M}}_{\boldsymbol{p}} \boldsymbol{n}\right)=c_{0} h^{(s)^{*}}\left(-\hat{\boldsymbol{M}}_{\boldsymbol{p}} \boldsymbol{n}\right)=h^{*}(-\boldsymbol{n})
$$

so that $H(\omega)$ also has zero-phase.

$\triangle \triangle \triangle$

Moreover, for the IIR case, if all the $p_{i}(n)$ 's are stable, it can be easily shown that the resulting $h(n)$ is also stable.

Here, we have to point out that this modified method doesn't apply to every $\boldsymbol{M}$ with large $J(\boldsymbol{M})$. For example, the matrix

$$
\boldsymbol{M}=\left[\begin{array}{rr}
10 & -1 \\
-1 & 10
\end{array}\right]
$$

with $J(\boldsymbol{M})=99$, does not have nonunit common factors among each column. Whenever this happens, we can use IFIR technique instead.

\section{CONCLUSIONS}

In MD multirate signal processing, filters with parallelepiped-shaped passbands governed by the decimation/ expansion matrix (an integer matrix) play an important role. In this paper, we have presented a method of designing such filters by starting with a proper 1D prototype filter and then using a simple transformation. These filters, although nonseparable, have separable polyphase components. Efficient polyphase implementations of these MD filters with complexity only proportional to a $1 \mathrm{D}$ filter are also presented. Important properties such as the Nyquist constraint, zero-phase constraint and BIBO stability can be easily achieved by using this method. We have shown several applications of the presented method, including many useful MD uniform DFT QMF bands and corresponding design examples. We have also generalized our method so that filters with an arbitrary parallelepiped-shaped passband (not necessary governed by an integer matrix) can be designed.

\section{REFERENCES}

11] D. E. Dudgeon and R. M. Mersereau. Mulhidimensional Digital Signal Processing. Englewood Clifts. NJ: Prentice-Hatl. 1984

12] C.-L. Lau and R. Ansari, "Two-dimensional digital filter design and implementation based on generalized decimation," in Proc. 20th Conf. Inform. Sci. Syst., Princeton, NJ, Mar. 1986, pp. 626-631.

[3] R. Ansari and C.-L. Lau. "Two-dimensional IIR filters for exact reconstruction in tree structured subband decomposition." Electrom. Letl. vol. 23, no. 12, pp. 633-634. June 1987.

14] R. Ansari, "Efficient IIR and FIR fan filters." IEEE Trams. Circuits Syst., vol. CAS-34, no. 8, pp. 941-945. Aug. 1987.

15] R. Ansari and S. H. Lee. "Two-dimensional nonrectangular interpolation, decimation, and tilter banks.." presented at the ICASSP 88. Apr. 1988.

16] R. H. Bamberger and M. J. T. Smith. "A filter bank for the directional decomposition of images: Theory and design." IEEE Trams. Signal Processing. vol. 40, no. 4, pp. 882-893. Apr. 1992

17] T. Chen and P. P. Vaidyanathan. "Multidimensional multirate filters derived from one-dimensional filters. "Electron. Le't,. vol. 27, no. 3. pp. 225-228. Jan. 1991.

[8] M. Vetterli, "Multidimensional subband coding: Some theory and algorithms." Signal Processing, vol. 6. no. 2. pp. 97-112. Feb. 1984.

$19 \mid$ E, Dubois. "The sampling and reconstruction of time-varying imagery with application in video systems." Proc. IEEE. vol. 73, pp. 502-522. Apr. 1985

$110 \mid$ G. Karlsson and M. Vetterli. "Theory of two-dimensional multirate filter banks," IEEE Trams. Acoust., Speceh, Signal Processing. vol. 38. no. 6. pp. 925-937. June 1990.

[11] P. P. Vaidyanathan. "Fundamentals of multidimensional multirate digital signal processing." Salhama, vol. 15, pp. 157-176. Nov 1990.

[12] E. Viscito and J. P. Allebach. "The analysis and design of multidimensional FIR perfect reconstruction filter banks for arbitrary sampling lattices," IEEE Trans. Circuits Syst. vol. CAS-38. no. 1. pp. 29-41, Jan. 1991.

113] T. Chen and P. P. Vaidyanathan. "The role of integer matrices in multidimensional multirate systems." IEEE Trams. Sighal Processing. vol. 41. no. 3. pp. 1035-1047. Mar. 1993. 
1141 T. Kailath. Linear Systems. Englewood Cliffs, NJ: Prentice-Hall, 1980.

[15] M. Vidyasagar, Control System Synthesis: A Factorization Approach. Cambridge, MA: M.I.T. Press, 1985

116] T. Chen and P. P. Vaidyanathan, "Least common right/left multiples of integer matrices and applications to multidimensional multirate systems." in Proc. IEEE Int. Symp. Circuits Syst.. San Diego. CA, May 1992, pp. 935-938

117] L. R. Rabiner and B. Gold, Theory and Application of Digital Signal Processing. Englewood Cliffs, NJ: Prentice-Hall. 1975.

[18] K. Swaminathan and P. P. Vaidyanathan, "Theory and design of uniform DFT, parallel, quadrature mirror filter banks," IEEE Trans. Circuits Sist., vol. CAS-33, no. 2. pp. 1170-1191. Dec. 1986

[19] P. P. Vaidyanathan. "Quadrature mirror filter banks, $M$-Band extensions, and perfect-reconstruction techniques, "IEEE ASSP Mag. . pp. 4-20, July 1987.

[20] T. Chen and P. P. Vaidyanathan, "Recent developments in multidimensional multirate systems," IEEE Trans. Video Technol., vol. 3, no. 2, Apr. 1993.

121] P. P. Vaidyanathan, "Design of doubly complementary IIR digital filters using a single complex all-pass filter, with multirate application." IEEE Trans. Circuits Syst., vol. CAS-34, no. 4. pp. 378-389. Apr. 1987

122] T. A. Ramstad, "IIR filter bank for subband coding of images, " in Proc. IEEE Int. Symp. Circuits Sist. Espoo. Finland, June 1988, pp. 827-830.

123] S. K. Mitra, C. D. Creusere, and H. Babic, "A novel implementation of perfect reconstruction QMF banks using IIR filters for infinite length signals," in Proc. IEEE lit. Symp. Circuits Syst.. San Diego. CA. May 1992. pp. 2312-2315.

[24] J. D. Johnston, "A filter family designed for use in quadrature mirror filter banks," in Proc. IEEE Int. Conf. Acoust. Speech, Signal Processing. Apr. 1980, pp. 291-294

[25] R. E. Crochiere and L. R. Rabiner, Malitate Digital Signal Processing. Englewood Cliffs. NJ: Prentice-Hall. 1983.

[26] P. P. Vaidyanathan. Multirate Systems and Filler Banks. Englewood Cliffs. NJ: Prentice-Hall, 1993.

[27] M. Renfors and T. Saramäki. "Recursive Nth-band digital filtersPart I: Design and Properties," IEEE Trans. Circuits Sy'st., vol. CAS34. no. l, pp. 24-39. Jan. 1987

[28] Y. Neuvo, C. Y, Dong, and S. K. Mitra, "Interpolated finite impulse response filters." IEEE Trans. Acoust. Speedh, Signal Processing. vol. 32. no. 3. pp. 563-570. June 1984

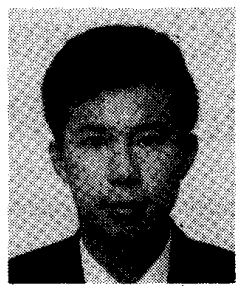

Tsuhan Chen ( $S^{\prime} 90$ ) was born in Taipei, Taiwan, Republic of China, on November 7, 1965. He received the B.S. degree in electrical engineering from the National Taiwan University, Taipei, Taiwan, in 1987, and the M.S. degree in electrical engineering from the California Institute of Technology in 1990 . He is currently working towards the Ph.D. degree in electrical engineering at the California Institute of Technology. His main research interests are in digital signal processing, multirate filter banks, and multidimensional mul-

tirate systems.

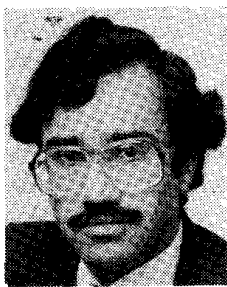

P. P. Vaidyanathan (S'80-M'83-SM' $88-F^{\prime} 91$ ) was born in Calcutta, India, on October 16, 1954. $\mathrm{He}$ received the $\mathrm{B}$. Sc. (Hons.) degree in physics and the $B$. Tech. and $M$. Tech. degrees in radiophysics and electronics, all from the University of Calcutta. India, in 1974, 1977, and 1979. respectively, and the Ph.D. degree in electrical and computer engineering from the University of California, Santa Barbara, in 198 ?

He was a postdoctoral fellow at the University of California, Santa Barbara, from September 1982 to March 1983. In March 1983 he joined the Department of Electrical Engineering of the California Institute of Technology as an Assistant Professor, and since 1988 has been as Associate Professor of Electrical Engineering there. His main research interests are in digital signal processing, multirate systems, wavelet transforms, and adaptive filtering.

Dr. Vaidyanathan served as Vice Chairman of the Technical Program Committee for the 1983 IEEE International Symposium on Circuits and Systems, and as an Associate Editor for the IEEE TrANSACTIONS ON CIR. CUITS AND SYSTEMS for the period 1985-1987. He also served as the Technical Program Chairman for the 1992 IEEE International Symposium on Circuits and Systems. He was a recipient of the Award for Excellence in Teaching at the California Institute of Technology for the year 1983-1984. He also received the NSF's Presidential Young Investigator Award in 1986. In 1989 he received the IEEE ASSP Senior Award for this paper on multirate perfect-reconstruction filter banks. In 1990 he received the $\mathrm{S}$. K. Mitra Memorial Award from the Institute of Electronics and Telecommunications Engineers, India, for his joint paper in the IETE journal. He was also the coauthor of a paper on linear-phase perfect reconstruction filter banks in the IEEE Transactions on Signal Processing, for which the first author (Truong Nguyen) received the Young Outstanding Author Award in 1993. He was elected a Fellow of the IEEE in 1991. 